\title{
In situ monitoring methods for selective laser melting additive manufacturing process based on images - A review
}

\author{
Bo Wu', Xiao-yuan Ji', * Jian-xin Zhou', Huan-qing Yang ${ }^{2,3}$, Dong-jian Peng ${ }^{2,3}$, *Ze-ming Wang ${ }^{4}$, Yuan-jie Wu', \\ Ya-jun Yin' \\ 1. State Key Laboratory of Materials Processing and Die \& Mould Technology, School of Materials Science and Engineering, Huazhong \\ University of Science and Technology, Wuhan 430074, China \\ 2. Xi'an Space Engine Co., Ltd., Xi'an 710100, China \\ 3. Center of National Defense Technology Industry Aerospace Special Components Additive Manufacturing Innovation, Xi'an 710100, China \\ 4. Nuclear Power Institute of China, Chengdu 610213, China
}

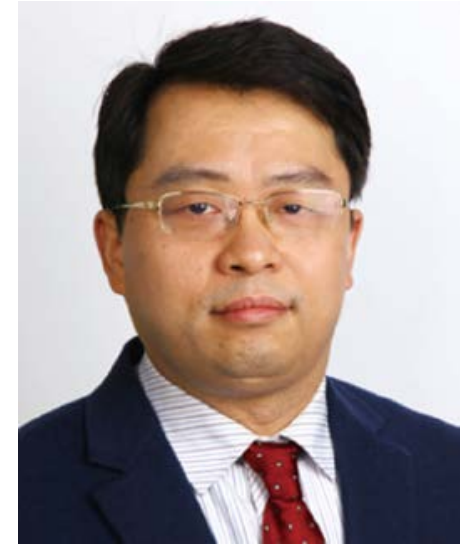

*Jian-xin Zhou

Born in 1975, Ph.D., Professor and doctoral supervisor. Prof. Zhou is the Vice Director of the State Key Laboratory of Materials Processing and Die \& Mould Technology, and the Chief Professor of the "Intelligent Green Casting Technology and Application" Cross Innovation Team. His research interests mainly focus on the digital casting (CAD/CAE/ ERP/MES/PDM/SCADA), intelligent casting and 3D printing (simulation and quality control). He has presided over the general projects of the National Natural Science Foundation, the High-grade CNC Machine Tool and Basic Manufacturing Equipment Project, the National High-Tech Research and Developement (863) Program of China, etc. Now, he is presiding over the National Key Research and Development Program of China: Network Cooperative Manufacturing and Intelligent Factory. In recent five years, he has published 129 SCI/EI/CSCD papers, and possesses 11 software copyrights and 12 invention patents. He was rewarded two National Science and Technology Progress Awards, ranked 2nd place in 2018 and 4th in 2007.

E-mail: zhoujianxin@hust.edu.cn

*Ze-ming Wang

E-mail:npicwzm@126.com

\begin{abstract}
Selective laser melting (SLM) has been widely used in the fields of aviation, aerospace and die manufacturing due to its ability to produce metal components with arbitrarily complex shapes. However, the instability of SLM process often leads to quality fluctuation of the formed component, which hinders the further development and application of SLM. In situ quality control during SLM process is an effective solution to the quality fluctuation of formed components. However, the basic premise of feedback control during SLM process is the rapid and accurate diagnosis of the quality. Therefore, an in situ monitoring method of SLM process, which provides quality diagnosis information for feedback control, became one of the research hotspots in this field in recent years. In this paper, the research progress of in situ monitoring during SLM process based on images is reviewed. Firstly, the significance of in situ monitoring during SLM process is analyzed. Then, the image information source of SLM process, the image acquisition systems for different detection objects (the molten pool region, the scanned layer and the powder spread layer) and the methods of the image information analysis, detection and recognition are reviewed and analyzed. Through review and analysis, it is found that the existing image analysis and detection methods during SLM process are mainly based on traditional image processing methods combined with traditional machine learning models. Finally, the main development direction of in situ monitoring during SLM process is proposed by combining with the frontier technology of image-based computer vision.
\end{abstract}

Key words: selective laser melting (SLM); forming process; images; in situ monitoring; molten pool region monitoring; scanned layer and powder layer monitoring

CLC numbers: TG221; Document code: A;

Article ID: 1672-6421(2021)04-265-21

\section{Significance of in situ monitoring during SLM process}

When SLM forming is conducted, a 3D digital model of the component is firstly sliced, discretized and dimension-reduced, and then the 0-dimensional parameters (spot diameter, laser power), 1-dimensional 
parameters (scanning speed), 2-dimensional parameters (scanning interval), layer height, and support mode, are set to generate the G code that can be read and executed by an SLM equipment. Secondly, the equipment executes the corresponding $G$ code of each layer and the metal powders are selectively melted and solidified "point by point, line by line and plane by plane" under the action of laser. When a layer is scanned, the working platform drops a height of a layer thickness along the Z-axis direction. When a new layer of metal powder is spread on the focal plane, the new powder layer is selectively melted and solidified rapidly, and fused with the former layer. The cycle is repeated until the component is formed ${ }^{[1-3]}$. To prevent oxidation during SLM process, the SLM process is completed in an inert gas environment. The process of SLM is shown in Fig. 1.

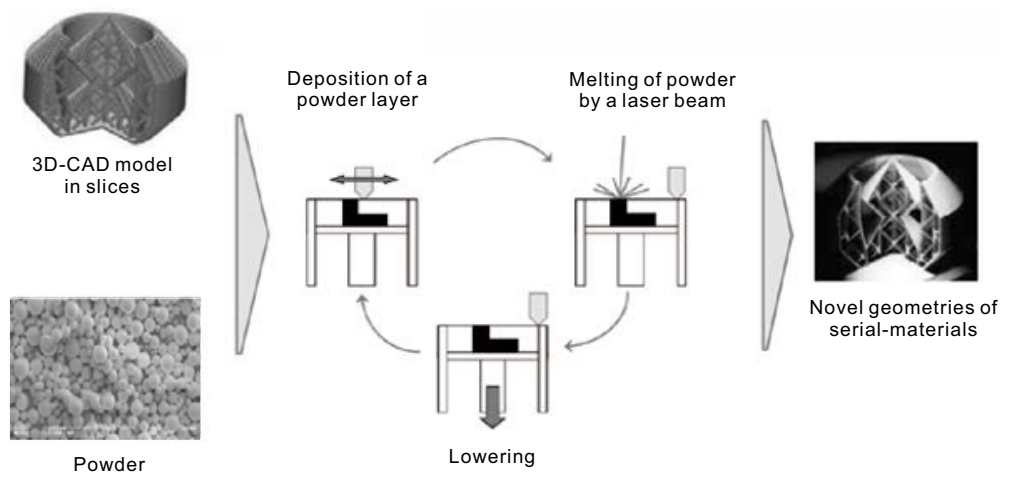

Fig. 1: Schematic diagram of SLM process ${ }^{[4]}$

A laser spot with a very small diameter is used as the energy source in SLM technology, and the method of "discrete-dimension-reduction" is adopted to simplify complex manufacturing, which causes SLM technology to possess the characteristics of small molten pool size, fast scanning speed and complicated melting-solidification process. The above characteristics of SLM forming technology, which cause the molten pool region splashing and the plume extremely unstable during the SLM process, eventually cause the quality fluctuation to be accumulated and magnified layer by layer. In severe cases, the SLM process will be interrupted, or even cause the scrap of the component ${ }^{[1-3]}$.

The poor quality stability of the formed components and the insufficient repeatability of SLM process have become the bottlenecks hindering further development, application and industrialization of SLM technology. Currently, the off-line inspection methods are mostly used to inspect the quality of components formed by SLM. However, off-line inspection has obvious hysteresis and does not allow the process parameters to be adjusted online to eliminate specific defects in time, which often leads to serious waste of materials, serious waste of time, and even delay of order delivery. To repair defects during SLM forming, SLM equipment operators are often required to be on constant duty so as to intervene timely when defects occur. However, the defect detection during SLM process based on human vision is susceptible to the physical and mental fatigue of workers, and it is difficult to achieve objective and accurate detection. Therefore, the in situ monitoring methods based on images during SLM process were developed to lay a foundation for improving the stability of quality of the component formed by SLM and the repeatability of the SLM process, and to provide quality diagnostic information for feedback control, which has become one of the research hotspots in the field in recent years ${ }^{[5,6]}$.

\section{Information sources of defects in SLM process}

Material melting, evaporation, chaotic movement, and jet of the molten metal in the melt pool will occur when a high-energy laser irradiates the powders. The recoater may vibrate or even get stuck when there are bulges on the powder bed. As shown in Fig. 2, these phenomena are accompanied by light, heat, sound, force, and other signals. There are two sources of visible light signal during SLM process. One is the LED light embedded in the building chamber. The other is the light emitted from the melt pool, spatters and plumes. The visible light could be detected and transferred into images by cameras. The heat signal mainly refers to infrared light. The detection of infrared light during SLM process mainly focuses on the infrared radiation emitted from the melt pool, spatters, plumes, scanned layer and powder coated layer. The heat signal could be detected and imaged by infrared (IR) or near-infrared (NIR) thermal imager. When the spatters and plumes are ejected from the melt pool, the acoustic signal is always caused by the pressure wave. The acoustic signal could be also generated when the powder-recoater recoats powder or collides with bulges in a layer. The microphone can be used to detect the acoustic signal. The thermal stress always generates the force signal. When the powder-recoater recoats powder, the force signal is also generated. The mechanical sensors are applied for the detection of the force signal generated during SLM process. The electrical signal is generated when the metal vapor is ionized into plasma. However, the electrical signal is too weak and includes noise. It is difficult to use the force signal and the acoustic signal to recognize defect type and grade. Therefore, visible light and infrared light are the two signals, which can reflect defects during the SLM process. These signals provide the most direct basis for in situ monitoring on quality, performance prediction and feedback control. The collection and analysis of the information mentioned above are important parts of in situ monitoring.

Table 1 shows the results of the literature according to the information generated during the SLM process and the corresponding detection targets. Figure 3 shows examples of various types of images captured during the SLM process in some literature. 


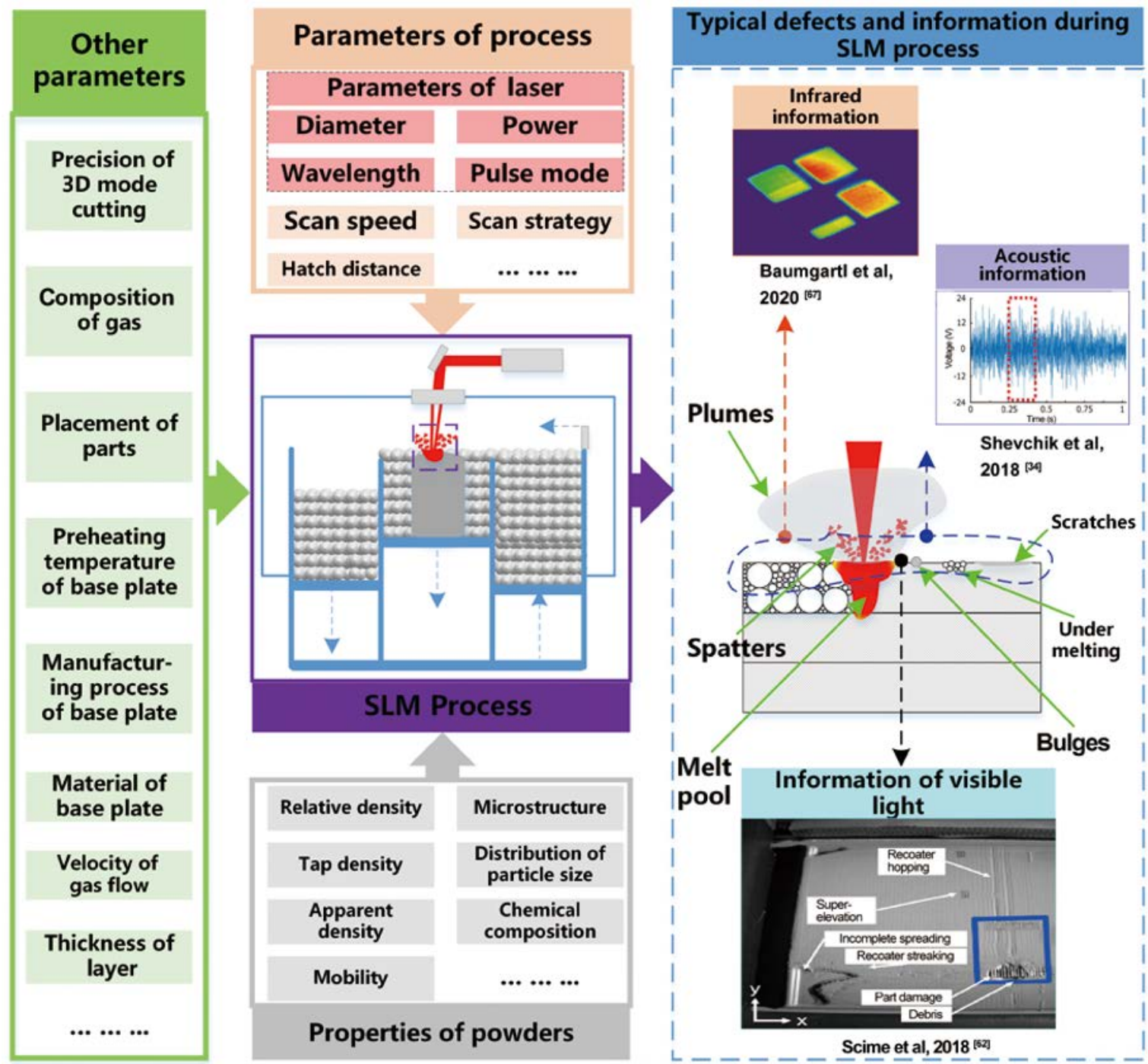

Fig. 2: Influencing factors, macro products, macro defects and information during SLM process

Table 1: Classification results of literature according to information generated during SLM process and corresponding detection objects

\begin{tabular}{|c|c|c|c|c|}
\hline \multirow{2}{*}{ Detection objects } & \multicolumn{4}{|c|}{ Information categories } \\
\hline & Visible light & Infrared light & Acoustic & Force \\
\hline $\begin{array}{l}\text { State of melt pool } \\
\text { and its adjacent space }\end{array}$ & $\begin{array}{c}{[15-17,27,37-39,56} \\
48-49,59,61,69]\end{array}$ & $\begin{array}{c}{[8-17,27,28-32,35-36,41,45} \\
54,59-60,64,66,68-69 \\
72-73,78]\end{array}$ & {$[33,34]$} & {$[74]$} \\
\hline $\begin{array}{l}\text { Morphology of scanned layer } \\
\text { and layer coated with powder }\end{array}$ & $\begin{array}{c}{[7,16,18-20,22-25,43} \\
46-47,50-52,62-63,70-71]\end{array}$ & $\begin{array}{c}{[21,26,42,44-46,57-58,67} \\
72-73,75-77]\end{array}$ & & \\
\hline $\begin{array}{c}\text { Temperature and } \\
\text { deformation along } z \text {-direction }\end{array}$ & & [40] & & {$[40]$} \\
\hline $\begin{array}{l}\text { Dynamics information generated } \\
\text { during SLM process }\end{array}$ & & & [53] & \\
\hline
\end{tabular}

\section{In situ monitoring methods for SLM process based on images}

As shown in Fig. 4, according to whether the sensor perceives information from the laser path, the in situ monitoring systems are divided into the coaxial in situ monitoring system and paraxial in situ monitoring system. Figure 5 presents the detection objects of the in situ monitoring system. In order to monitor different types of objects, various of in situ monitoring systems for SLM process are developed in this field, as shown in Fig. 6. 


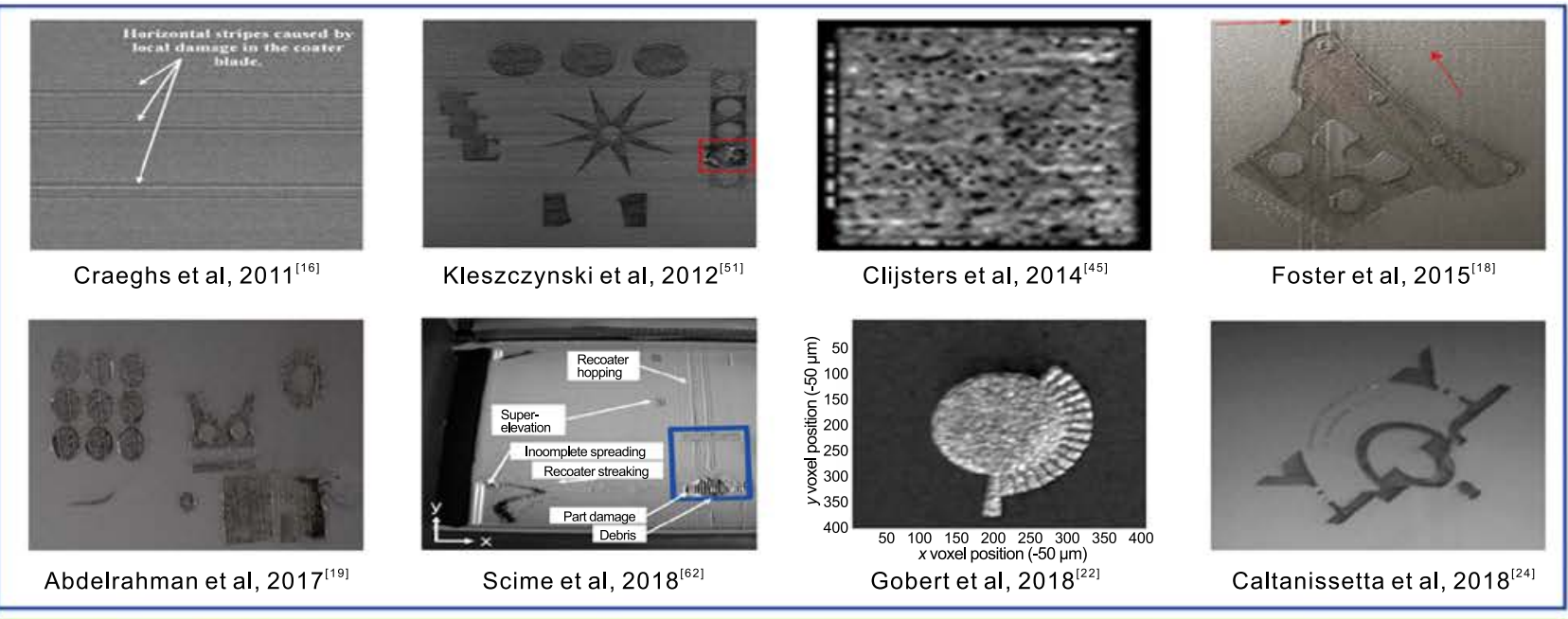

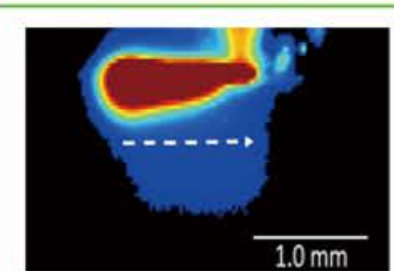

Gould et al, $2021^{[10]}$

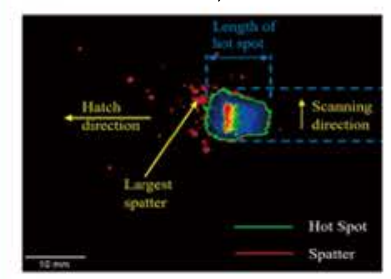

Yakout et al, 2020[12]

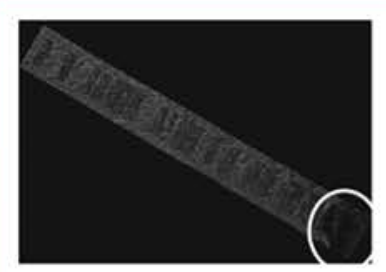

Craehgs et al, $2012^{[17]}$

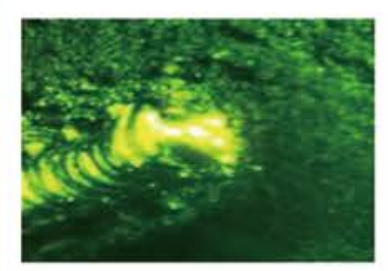

Alkahari et al, 2014

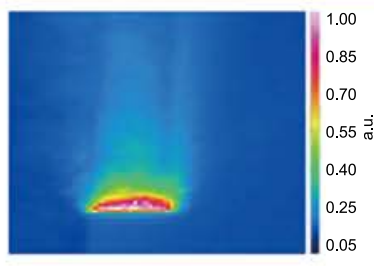

Krauss et al, $2012^{[75]}$

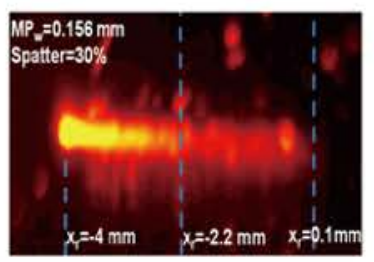

Yang et al, $2020^{[68]}$

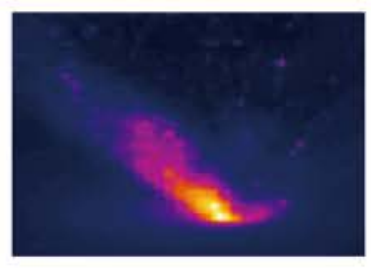

Grasso et al, 2018 $8^{[35]}$

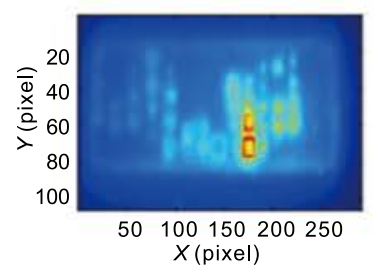

Elwarfalli et al, 2019 $9^{[11]}$

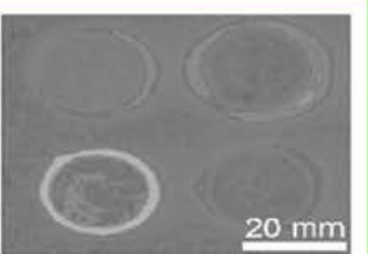

Bartlett et al, 2018 $[21]$

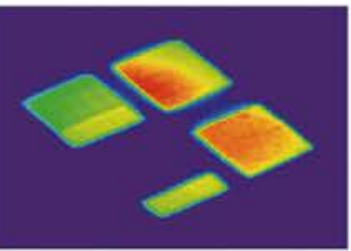

Baumgartl et al, $2020^{[67]}$

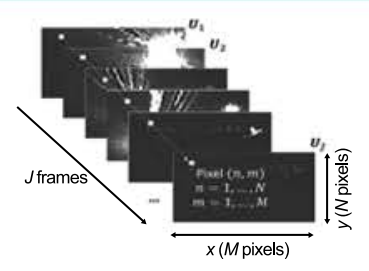

Grasso et al, $2017^{[38]}$

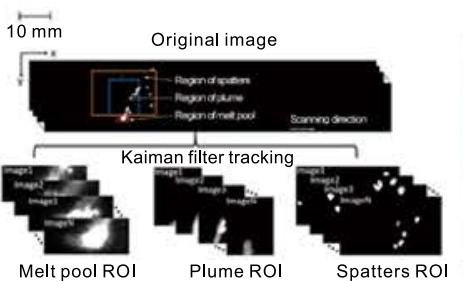

Zhang et al, $2018^{[31]}$

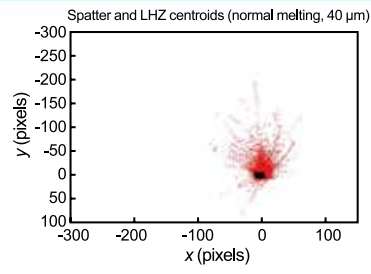

Repossini et al, $2017^{[37]}$

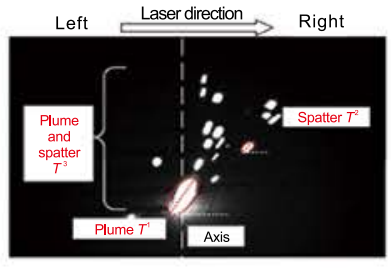

Ye et al, $2018^{[29]}$

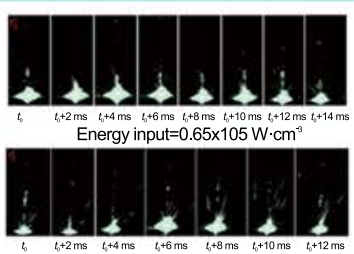

Wang et al, $2017^{[14]}$

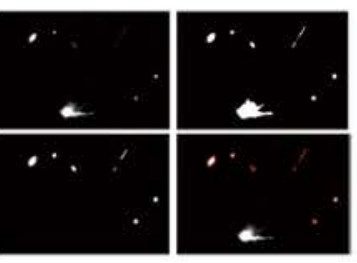

Yang et al, $2020^{[9]}$
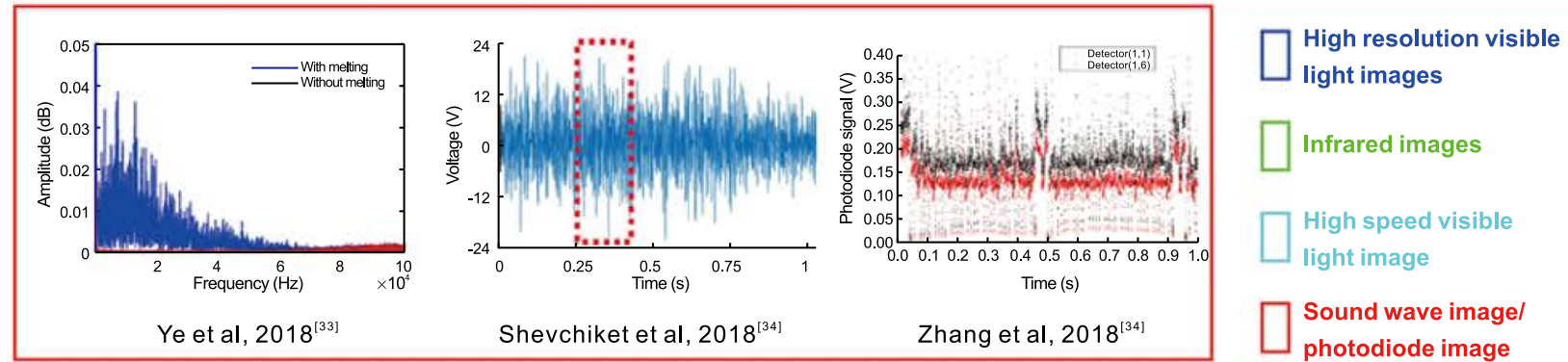

Fig. 3: Examples of various kinds of images captured during SLM process from some literatures 
(a)

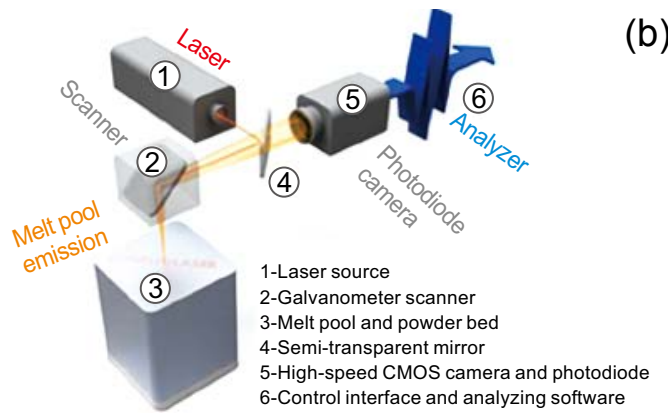

(b)

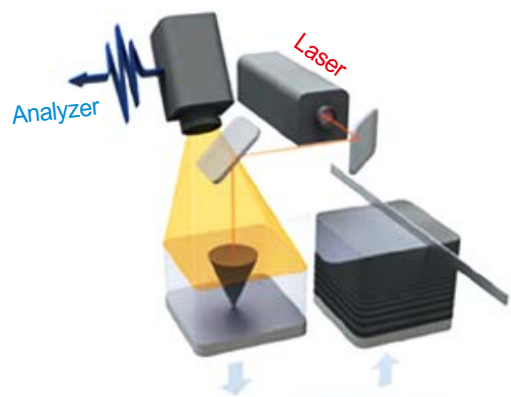

Fig. 4: Schematic diagram of in situ monitoring system during SLM process ${ }^{[79]}$ : (a) coaxial in situ monitoring system; (b) paraxial in situ monitoring system

(a)

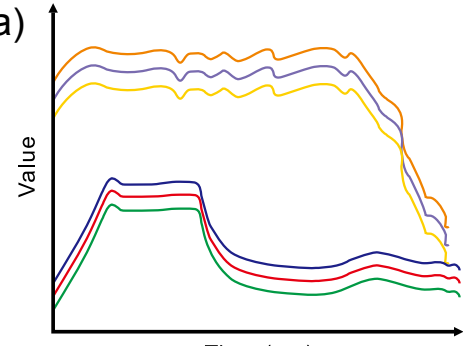

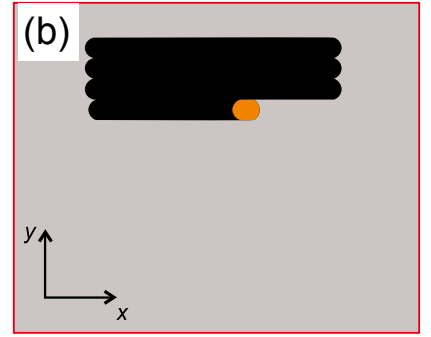

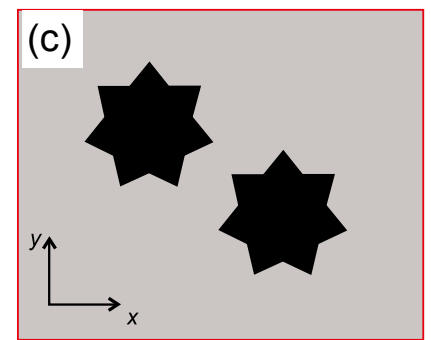

Fig. 5: Detection objects during SLM process: (a) one-dimensional time sequence information (such as temperature, sound, atmosphere pressure, etc.) ${ }^{[80]}$; (b), (c) 2D spatial information (such as melt pool image, scanned layer image, image of layer coated with powder and infrared image, etc. ${ }^{[81]}$

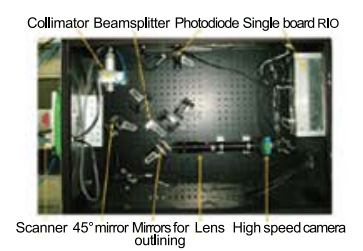

Coaxial high speed camera ${ }^{[1]]}$

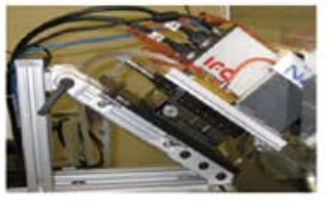

High speed infrared camera ${ }^{[6]]}$

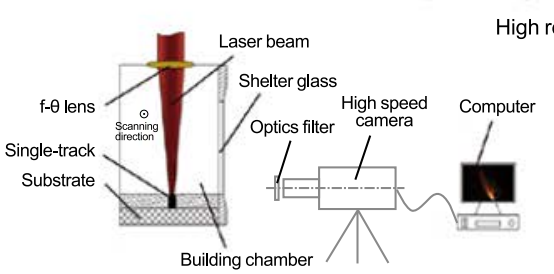

High speed camera ${ }^{[14]}$

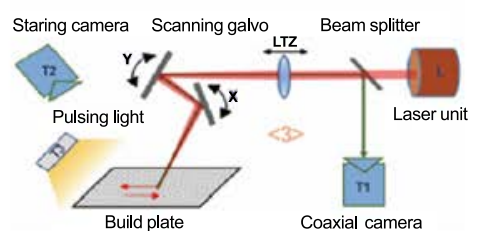

Coaxial high speed camera ${ }^{[8]}$

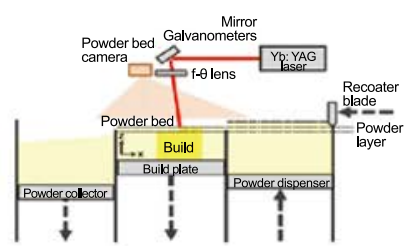

High resolution camera ${ }^{[62]}$

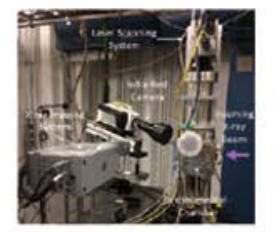

High speed infrared camera ${ }^{[10]}$

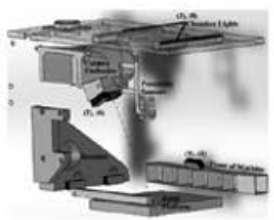

High resolution camera ${ }^{[22]}$

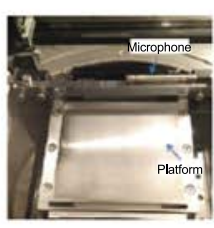

Microphone ${ }^{[33]}$

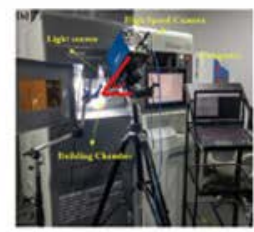

High speed camera ${ }^{[9]}$

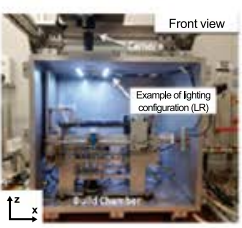

High resolution camera ${ }^{[24]}$

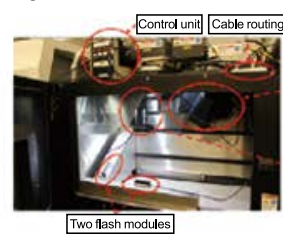

High resolutin camera ${ }^{[19]}$

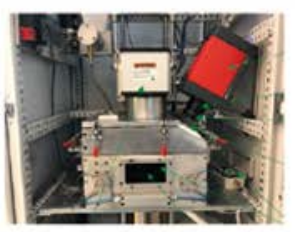

High speed infrared camera ${ }^{[12]}$

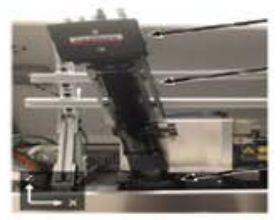

High speed camera ${ }^{[64]}$

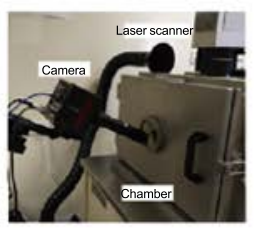

High speed camera ${ }^{[22]}$

Fig. 6: Literature examples of image acquisition devices and schematic diagram during SLM process

Compared with acoustic, electrical, mechanical and other signals generated during SLM process, optical signals conforming to human intuitive visual perception can be transformed into digital images by various cameras, which provides richer and more intuitive detection information and quality traceability information. Therefore, most of the in situ monitoring methods reported in literature are based on images. The detection objects include the melt pool and its byproducts, and the powder coated layer and scanned layer. The captured images are divided into the infrared thermal images and the visible light images according to the information of different light wave bands. 


\subsection{In situ monitoring of melt pool and its byproducts based on visible light imaging}

Craeghs et al. ${ }^{[15-17]}$ adopted the strategy of coaxial in situ monitoring, and used a large-area planar photodiode and a high-speed camera to capture images of the melt pool area at a high frequency during the SLM process ${ }^{[15-17]}$. A real-time image processing method was also developed. By sampling and recording the location and the data of the melt pool at the same time, a data representation method that maps the data of the melt pool to the X-Y plane was also developed and used to detect deformation due to thermal stress and overheating ${ }^{[27]}$. With the signal of the photodiode being analyzed, the dynamic relationship between the laser power and the photodiode signal was determined, and a feedback controller with the optimal bandwidth was designed based on this dynamic relationship ${ }^{[49]}$.
The anomaly detection method based on data representation does not seem to be with high accuracy. What is more, the feedback control mentioned may simply stop the process when an anomaly is detected, and the feedback controller may not be able to repair the defects generated during SLM process, but it created a precedent for the follow-up research in this field.

Figure 7 presents the device, images, image processing methods and results of the paraxial in situ monitoring of melt pool during SLM process. Grasso et al. ${ }^{[37]}$ used a high speed visible light camera shown in Fig. 7(a) to collect images along the laser scanning path, and extracted different statistical descriptors for images of spatters [Fig. 7(j)]. In addition, three logistic regression models were respectively fitted to three data sets (data set only containing laser heating zone, data set not only containing the information of spatters, and data set
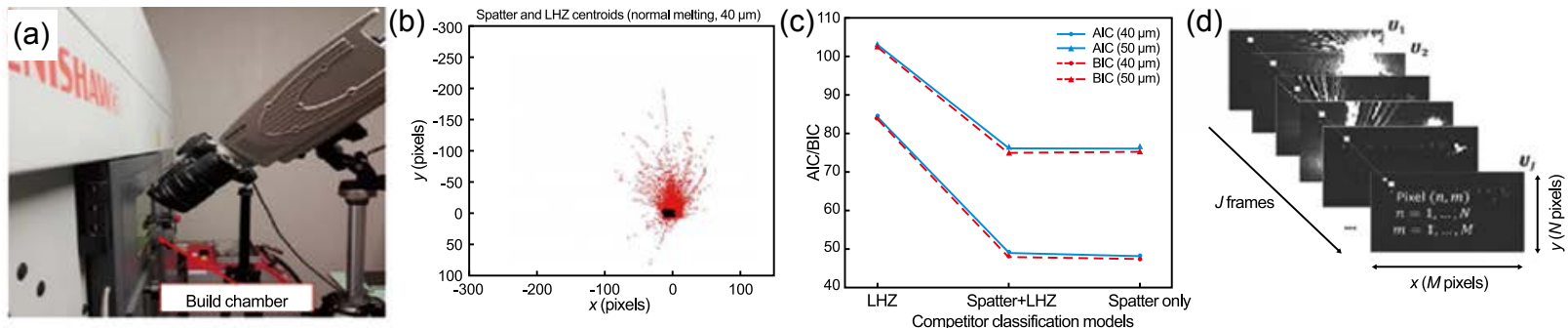

(e)

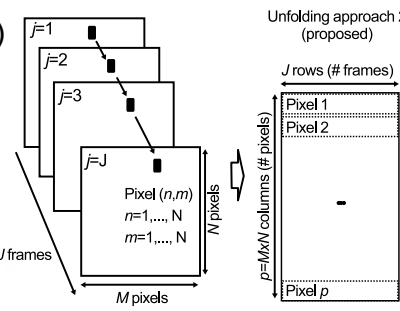

(i)

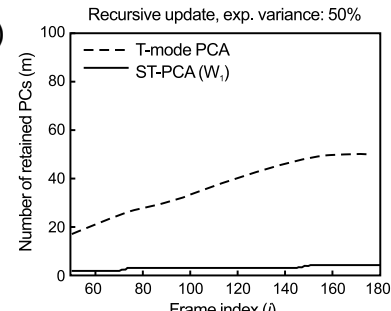

(k)

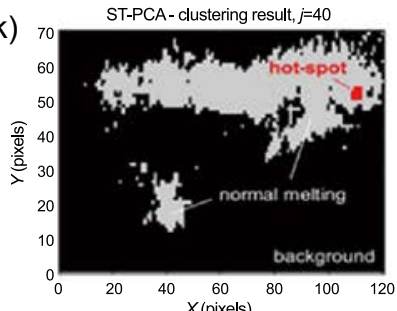

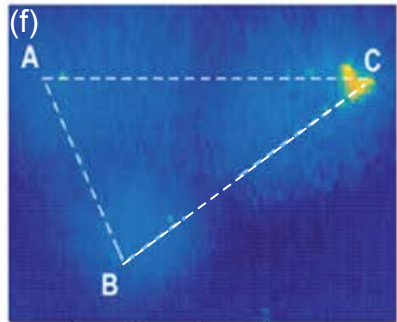

(g)

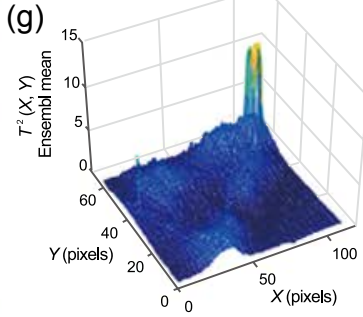

(h)

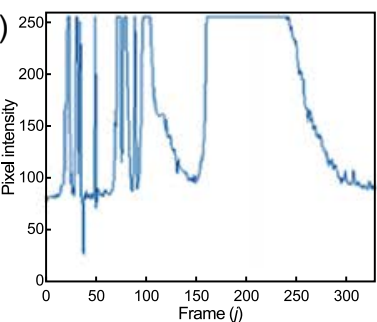

(j)
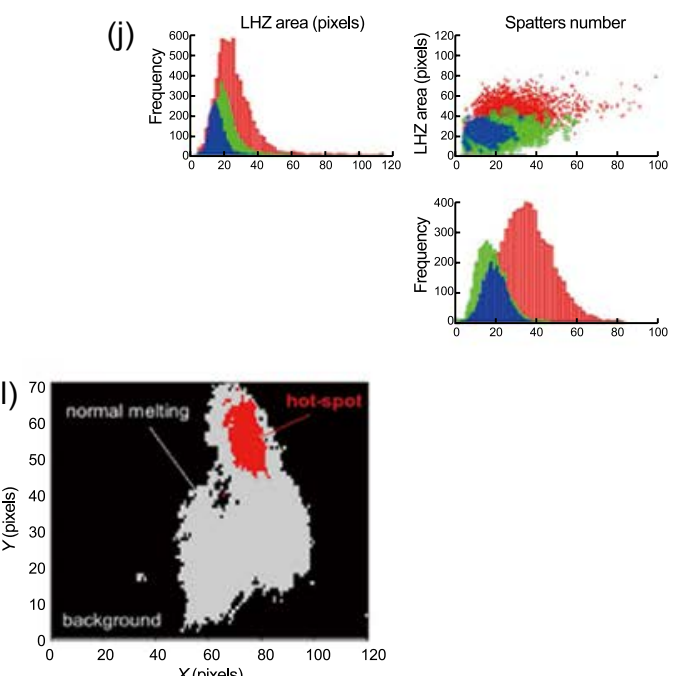
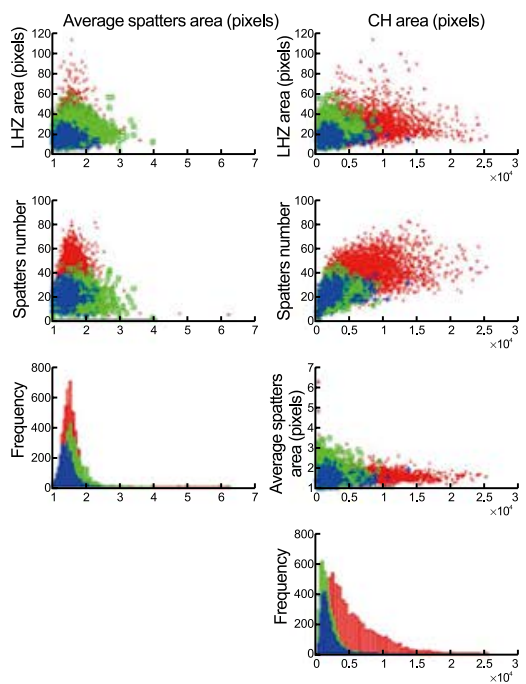

Fig. 7: Paraxial in situ monitoring of state of melt pool during SLM process: (a) schematic diagram of monitoring system of melt pool and by-products (spatters and plumes) based on high speed visible light imaging ${ }^{[37]}$; (b) melt pool and spatters under normal melting conditions ${ }^{[37]}$; (c) Akagi information criterion values and Bayesian information criterion values of classification models with and without splash information ${ }^{[37]}$; (d) visible light images stream during SLM process ${ }^{[33]}$; (e) schematic diagram of the image stream converted to a pixel-related form ${ }^{[38]}$; (f) and (g) defect areas detected using developed statistical methods ${ }^{[38]}$; (h) grayscale changes of local "hot spots" in each frame ${ }^{[39]}$; (i) time consuming curve (solid line) of proposed spatially weighted PCA algorithm ${ }^{[39]}$; (j) scatter plots of four statistical descriptors for spatters in different melting states ${ }^{[37]}$; (k) and (I) detection results based on spatially weighted PCA clustering segmentation ${ }^{[39]}$ 
only containing the information of spatters $)^{[37]}$. As shown in Fig. 7(c), the capacity and fitting accuracy of the three models are evaluated using Akaike Information Criterion and Bayes Information Criterion. Comparative analysis demonstrates that the feature of spatters improves the performance of melting state recognition during SLM process, which seems to be consistent with the common sense in data science that more useful and relevant features always lead to higher accuracy. As shown in Fig. 7(e), a pixel-related image stream conversion method for PCA was proposed ${ }^{[38]}$. A statistical descriptor based on principal component analysis (PCA), which was used to process the images, can identify the defect area in the image [Figs. 7(f) and $(\mathrm{g})]^{[38]}$. Finally, automatic defect detection was realized by using an image segmentation method based on clustering. To further improve the performance of defects detection during the SLM process of zinc powder, Marco et al. ${ }^{[39]}$ proposed a segmentation method based on a spatial weighted PCA to represent the temporal correlation and spatial location information correlation of pixel intensity of each frame of video. The comparative analysis demonstrates that this method can detect defects faster than T-mode PCA during the SLM forming process of zinc powder [Figs. $7(\mathrm{k})$ and (1)]. The camera for image acquisition is placed outside the building chamber on a tripod, which means that the angles and placement are not exactly the same each time. Therefore, the hyperparameters of the algorithm need to be tuned in advance.

Ji et al. ${ }^{[61]}$ adopted the in situ monitoring system to collect the images of spatters during the forming process. The description operator in polar coordinates for the spatters in the image was designed to extract features of spatters, which can be used to distinguish different energy densities. The description operator is an effective feature to recognize different level of energy densities.

As shown in Figs. 8(a) and (b), Scime et al. ${ }^{[64]}$ modified the EOS M290 camera and added a high speed camera to capture the images that can reflect the melt pool topography [Fig. 8(c)]. As shown in Fig. 8(d), the images were firstly converted into coaxial form through image transformation algorithm, then, the visual word bags based on SIFT (Scale-Invariant Feature Taransform) and HOG (Histogram of Oriented Gradient) were employed to extract the image features [Figs. 8(e) and (g) $]^{[64,66]}$. The corresponding labels were obtained by observing the actual microscopic morphology of melt pool [Fig. 8(f)] ${ }^{[65]}$. Finally, a data set for training the SVM model was constructed. The distribution of the data set after T-SNE processing is shown in Fig. 8(h) ${ }^{[66]}$. The dimension of the features of images extracted by the Visual Word Bags method is high, which means that PCA should be applied on the data set before T-SNE to visualize the intrinsic 2-D distribution of the data set. The distribution of the data set after T-SNE processing does not seem to have high discrimination, but the method has been proved to be feasible to recognize the melt pool defects.

\subsection{In situ monitoring of melt pool and its byproducts based on infrared imaging}

As shown in Figs. 9(a) and (b), Ye et al. ${ }^{[29]}$ used the paraxial near infrared high speed imaging system to capture the images of melt pool, spatters and plumes during SLM process [Fig. 9(c)], and obtained low resolution images $(100 \times 125)$ by downsampling. The low-resolution images were directly used as the input of the convolutional neural network (CNN) or as the input of the improved deep confidence network, respectively, to realize the melting state recognition during the SLM process. It was found that the improved deep confidence network needed less signal preprocessing, less parameter selection, and less feature extraction. The accuracy of identification of five melting states reached $83.40 \%$, which is not a satisfying result, and the improved deep confidence network may be a little underfitting. When the architecture is designed effectively and the hyperparameters are tuned, convolutional neural network should perform much better than deep confidence network, which has been proved in their follow-up research.
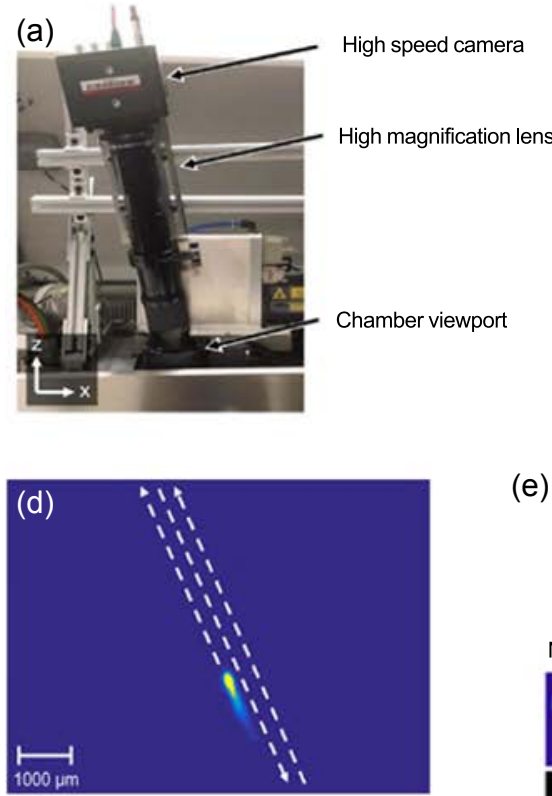

(b)

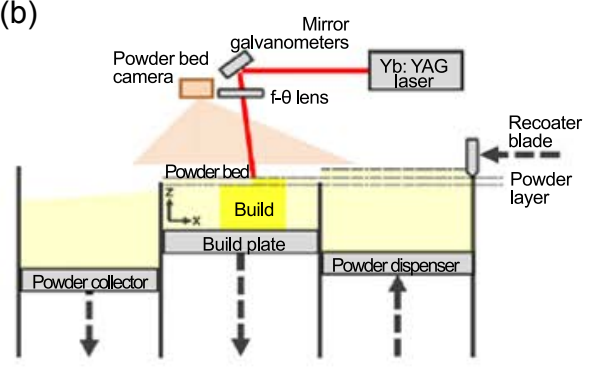

No contrast adjustment

(e) Reduced contrast

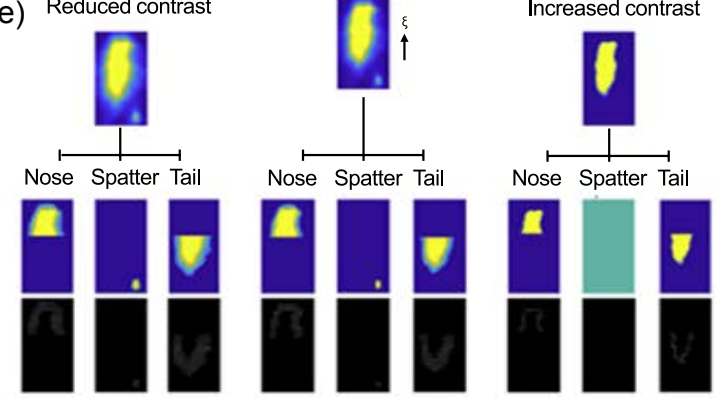

(c)

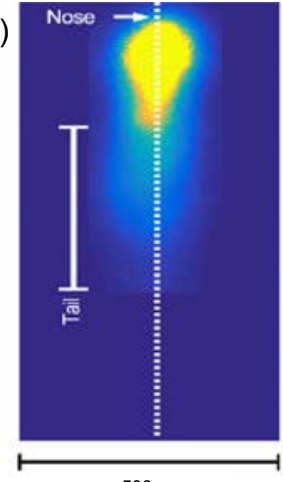

$500 \mu \mathrm{m}$ 


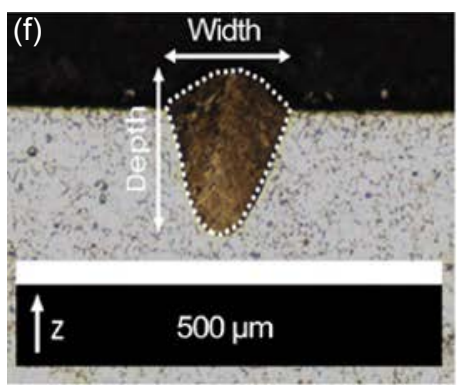

(h)

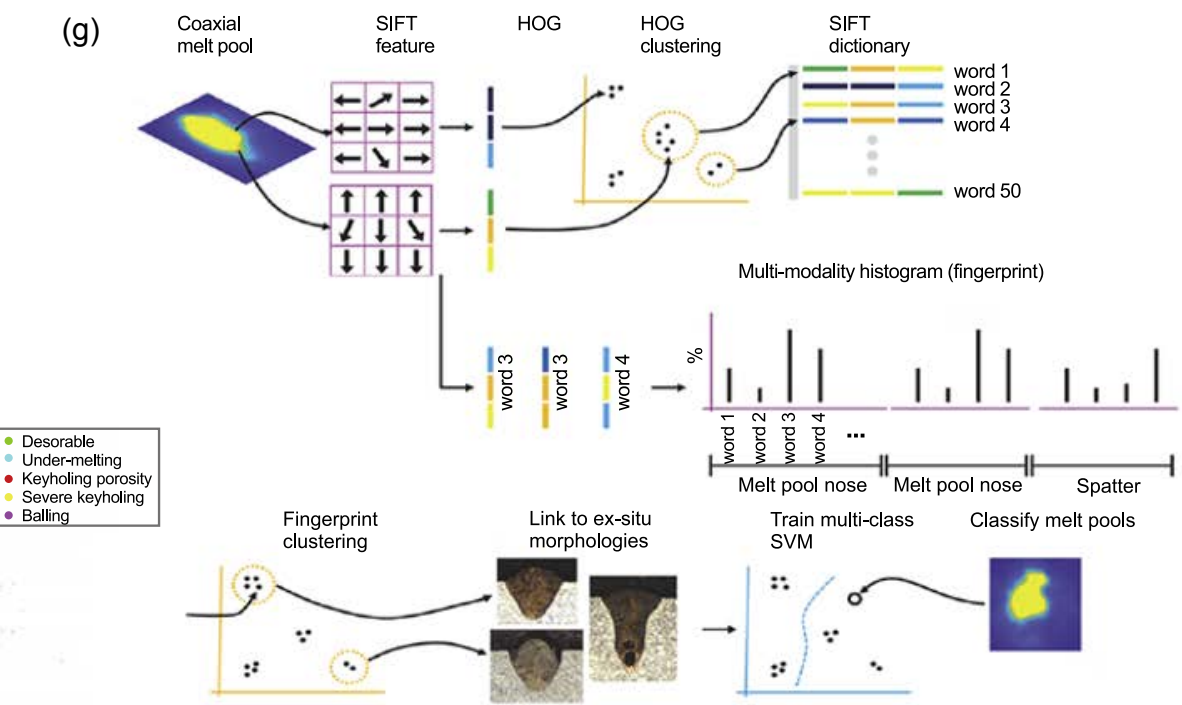

Fig. 8: Melt pool morphology acquisition system based on high speed camera ${ }^{[64,66]}$ (a and b), image of melt pool captured by high speed camera ${ }^{[64]}$ (c), image of melt pool after coordinate transformation ${ }^{[64]}$ (d), color enhanced image of melt pool and the corresponding SIFT feature extraction results ${ }^{[6]]}(\mathrm{e})$, example of actual microstructure of melt pool ${ }^{[65]}(\mathrm{f})$, schematic diagram of melt pool defect classification algorithm based on visual word bag and $\operatorname{SVM}^{[66]}(\mathrm{g}), 2 \mathrm{D}$ T-SNE distribution of data set constructed by visual word bags ${ }^{[66]}(\mathrm{h})$

Ye et al. ${ }^{[30]}$ also designed single-track-scanning experiments with different laser powers or scanning speeds. The paraxial near-infrared high-speed imaging system shown in Figs. 9(a) and (b) was employed to obtain images of melt pool, spatters and plumes under corresponding process parameters. The statistical features such as area, orientation of the main axis of the outer ellipse, circumference, length, width, and brightness were extracted by image processing method. The variation law of the statistical features of the image with the process parameters was studied. It was found that the features of plumes and spatters extracted from the images were significantly different under different melting conditions. In addition, the influence of process parameters on the width of melt track was studied [Figs. 9(e), (f)] ${ }^{[29-30]}$, which proved the feasibility of using plume-splash characteristics to identify melting state during SLM process. The similar conclusion that the feature of the spatters improves the performance of melting state recognition during SLM process has been also presented in the research of Grasso et al. ${ }^{[37]}$. This provides a theoretical basis for melting state detection.

Zhang et al. ${ }^{[31]}$ also adopted the system shown in Figs. 9(a) and (b). After adjusting the angle between the camera and the powder bed plane [Figs. 9(h), (i)], the images of melt pool, spatters and plumes during SLM process were captured again [Fig. 9(j)]. The sub-images were obtained from each frame by using the Kalman filter algorithm. The statistical features of melt pool, spatters and plumes were extracted based on the domain knowledge of SLM. After dimension reduction through PCA, a support vector machine model was trained, and achieved a $90.1 \%$ accuracy for the three melting states. What is more, Ye et al. also trained a CNN with an accuracy of $92.7 \%$, which demonstrated that $\mathrm{CNN}$ was a promising model for in situ monitoring during SLM process. The CNN model may be a little underfitting. The accuracy should be higher when the architecture is designed effectively and the hyperparameters are tuned. What is more, there should be no doubt that the deep CNN models perform better than SVM on the image recognition task.

Based on the research ${ }^{[30]}$, Zhang et al. ${ }^{[32]}$ developed a new image processing method to extract the sub-images containing melt pool, spatters, and plumes. The features, such as the brightness of melt pool, the area of plumes, the orientation of plumes, the quantity of spatters, the area of spatters, the orientation of spatters and the speed of spatters, were extracted from images. The correlation between four different scanning qualities and the extracted features was studied. It was found that the features extracted by the image processing method could be used as the potential index of SLM quality evaluation. However, the features extraction method is based on the domain knowledge, which makes the massive data generated during SLM process underutilized. To accurately recognize the melt states based on images, the massive data generated during SLM process should be fully applied to develop the method for feature extraction.

As shown in Fig. 10(a), Grasso et al. ${ }^{[35]}$ used the in situ monitoring system to capture the images [Fig. 10(b)] during the SLM process of zinc powder. The method to extract features of plumes [Figs. 10(c), (d)] was combined with data mining to implement the inspection, which demonstrated the applicability of features of plumes for distinguishing the state during SLM process. Based on the domain knowledge and experimental research, the data-driven automatic alarm rules were designed, and the in situ monitoring of melt stability during SLM process of zinc powder was implemented. As 

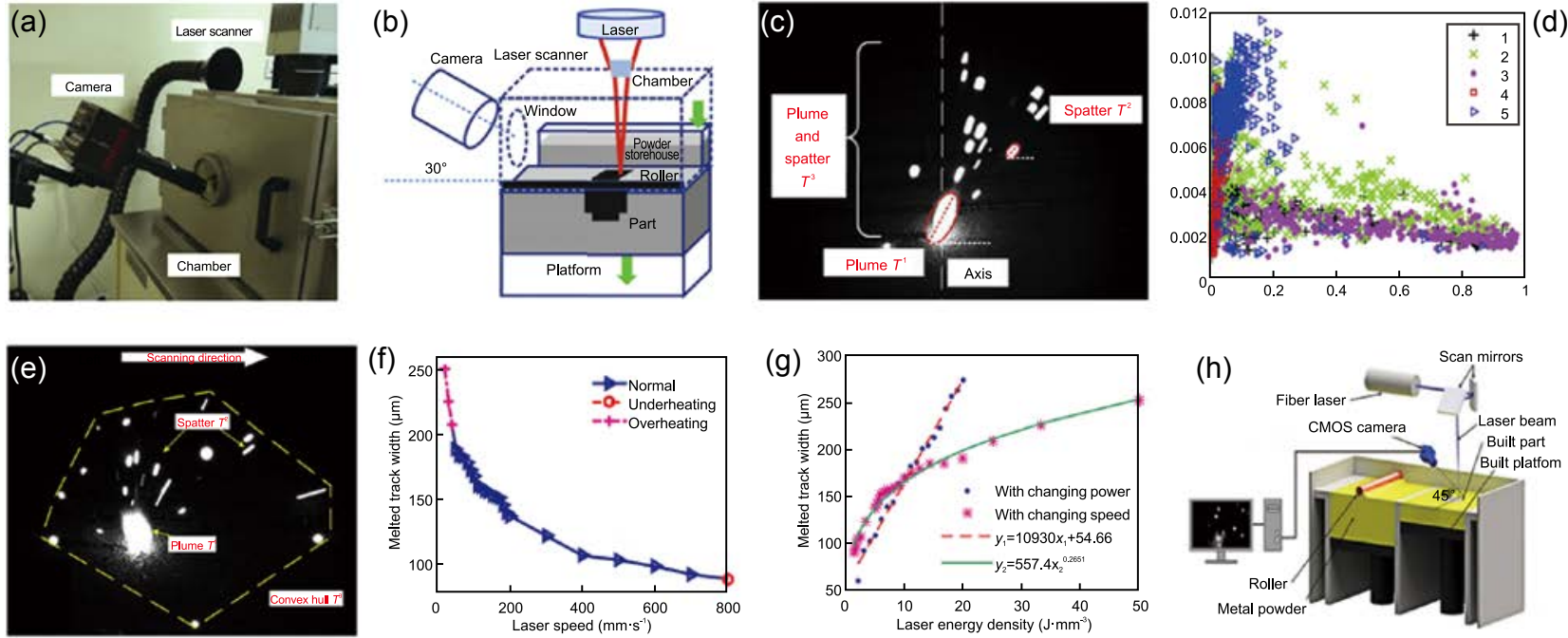

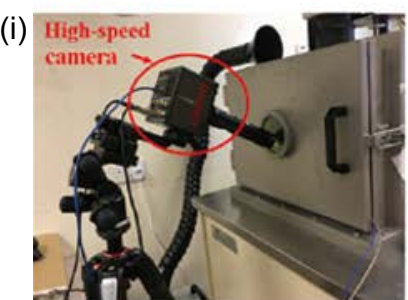

(j)
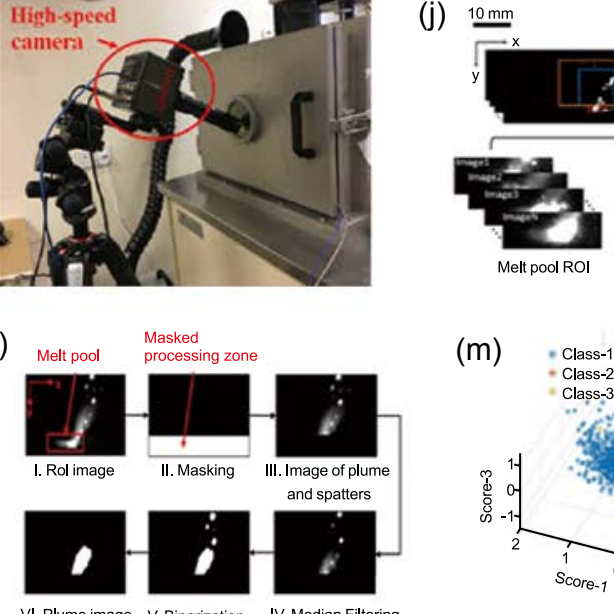

(o)
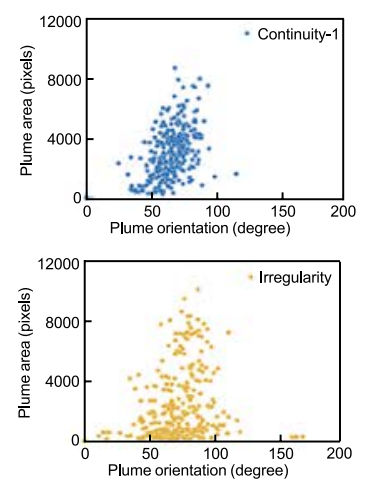

(m)
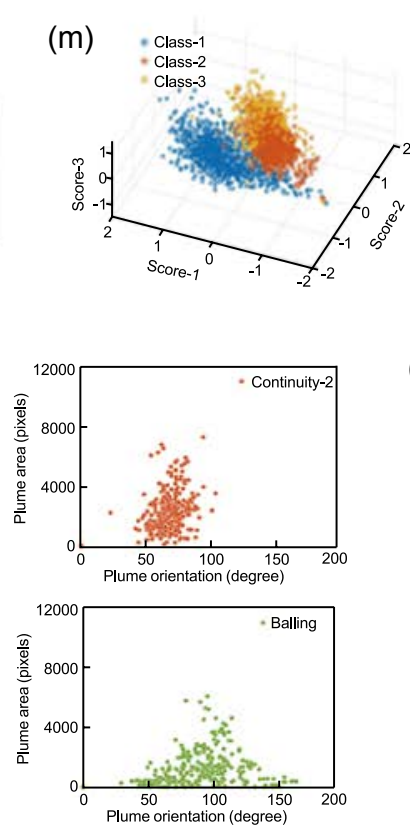

(k)

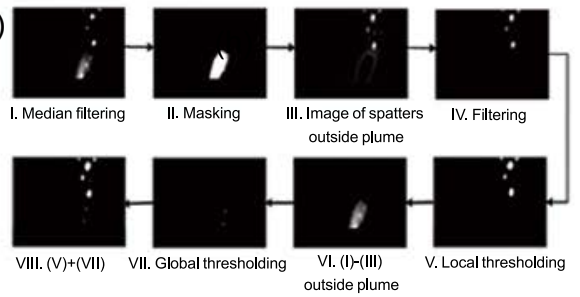

Verification result of CNN model

(n)
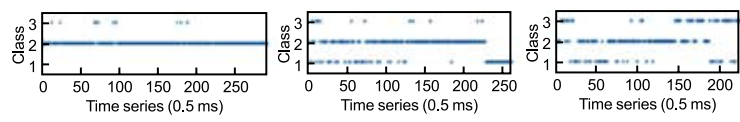

Verification result of SVM model
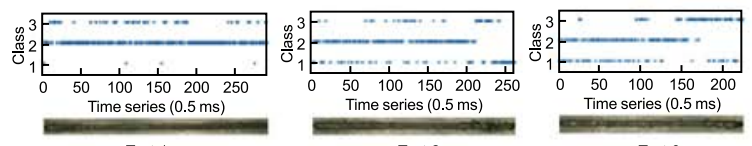

Test-3

(p)
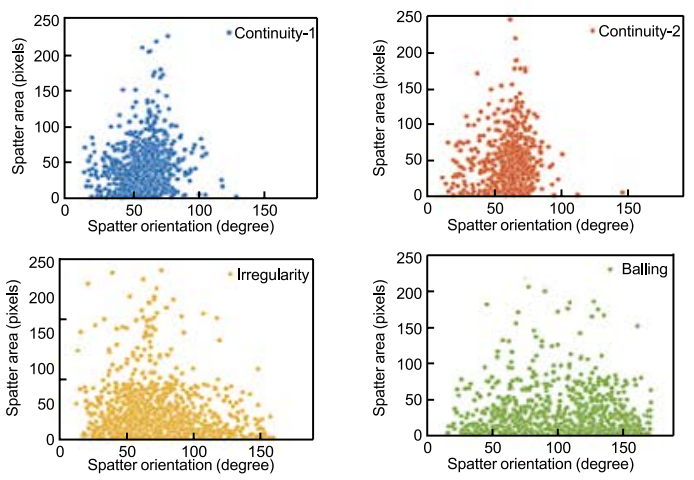

Fig. 9: In situ monitoring of melt pool, spatters and plumes during SLM process based on near infrared high speed imaging: (a), (b) in situ monitoring system (the angle between camera and powder bed is $30^{\circ}$ ) ${ }^{[29]}$; (c), (e) images of spatters

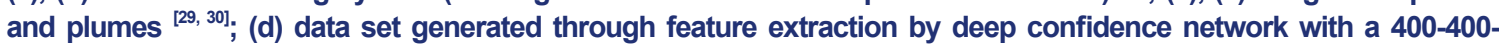
400-400 structure ${ }^{[29]}$; (f) curve of width of melt pool changing with scanning speed ${ }^{[30]}$; (g) curve of width of melt pool changing with energy density ${ }^{[30]}$; (h) and (i) in situ monitoring system (angle between camera and powder bed is $\left.4^{\circ}\right)^{[31]}$; (j) original image and images of melt pool, spatters and plumes obtained by Karman filtering ${ }^{\left[{ }^{31]} \text {; }\right.}$ (k) flow chart of method of plumes extraction ${ }^{[32]}$; (I) flow chart of method of spatters extraction ${ }^{[32]}$; (m) first three principal components after PCA dimension reduction of data set containing all features ${ }^{[31]}$; $(n)$ verification results of recognition ability of convolutional neural network and support vector machine ${ }^{[31]}$; (o) statistical results of plume orientation and area of four states ${ }^{[32]}$; $(p)$ statistical results of spatters orientation and area of four states ${ }^{[32]}$

shown in Fig. 10(e), Grasso et al. ${ }^{[36]}$ proposed a new plume feature description method, in which the plume periphery was fit with ellipse to represent the angle of the plumes. As shown in Fig. 10(f), the plumes and spatters were classified by support vector machine. The angle, gray scale and area of the plumes were used as the descriptors, and the scatter plot is shown in Figs. 10(g) and (h). Finally, the multi-mode control diagram based on SVM was used to monitor the stability during SLM 
(a)

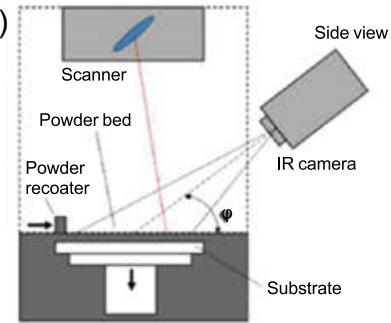

(e)

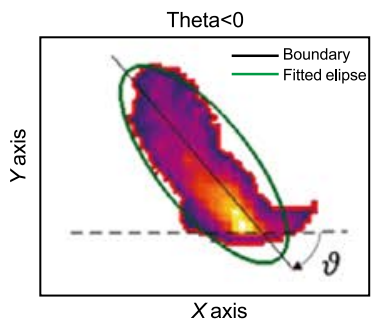

(b)

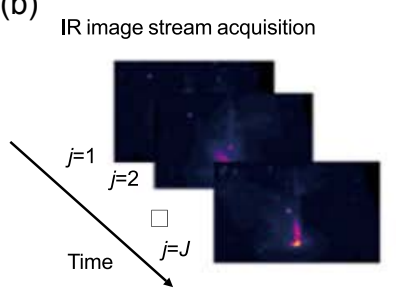

(f)

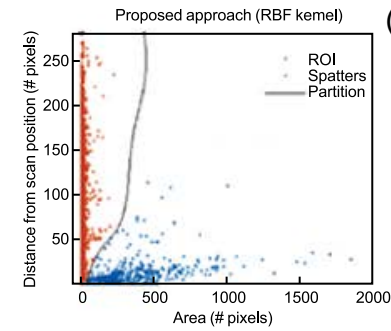

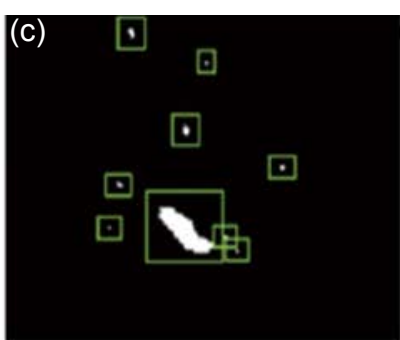

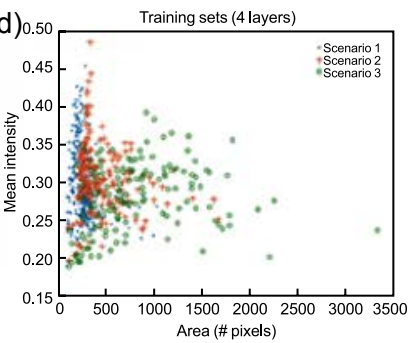

(g)

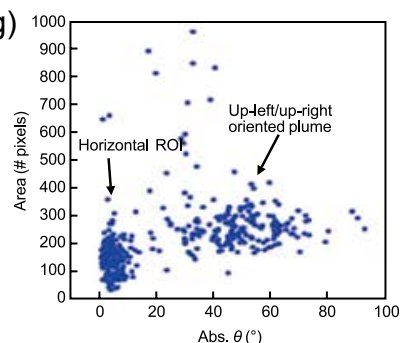

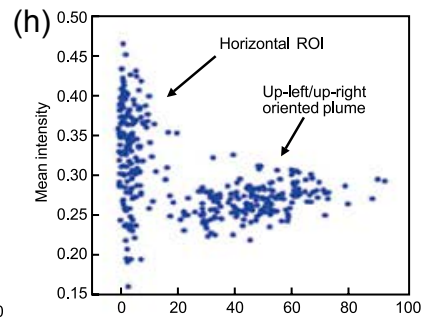

Fig. 10: In situ monitoring during SLM process: (a) schematic diagram of in situ monitoring system of melt pool and by-products (spatters and plumes) based on high speed infrared imaging ${ }^{[35]}$; (b) example of infrared image ${ }^{[35]}$; (c) image after segmentation ${ }^{[35]}$; (d) scatter plot of area and gray value of plumes extracted from image ${ }^{[35]}$; (e) circumscribed ellipse of plume ${ }^{[36]}$; (f) classification results of support vector machine ${ }^{[36]}$; $(\mathrm{g})$ and $(\mathrm{h})$ scatter plots of dip angle, area and gray level of plume ${ }^{[36]}$

process at a faster speed. Although the performance of the proposed methods seems to be good, the features extracted from images are low level and have no semantic information. If the deep neural network is applied to extract high level semantic information, the performance to monitor the stability during SLM process would be better.

Foster et al. ${ }^{[58]}$ used the infrared imaging system shown in Fig. 11(a) to measure the infrared radiation intensity of the laser-material interaction area in both space and time dimensions [Figs. 11(b) and (c)], and found that higher infrared radiation intensity and lower surface roughness, lower porosity, and coarser solidified grain structure were closely related. The result of this research work has important theoretical guiding significance. However, if some machine learning models or deep learning models are trained on the large amounts of image data sets, the data set with large amounts of images is utilized as it should be.

Forien et al. ${ }^{[59]}$ used an in situ monitoring system to inspect the forming process of the $316 \mathrm{~L}$ stainless steel single-track under different laser powers and scanning speeds. The defects characterized by X-ray imaging were associated with the thermal signals monitoring. Then, the probability of defect generated was calculated. It was found that the prediction of hole defects could be implemented by high-speed and high-temperature in situ monitoring of melt pool during the SLM process. Zouhri et al. ${ }^{[60]}$ used the melt pool radiation in situ monitoring system to collect the radiation information of the melt pool region during the SLM process, and then conducted a correlation study with the density of the formed part. A description operator for infrared thermal signals was developed, based on which multiple support vector machine models for predicting the three types of density levels were trained. Compared with deep neural networks, the support vector machine demonstrated a better generalization ability for this problem. The reason why the SVM performs better than deep neural networks may be that the data set is small, and the deep neural networks are over fitted. When there are more samples in the data set, the deep neural networks may demonstrate better performance. Transfer learning may also improve the performance of deep neural networks.
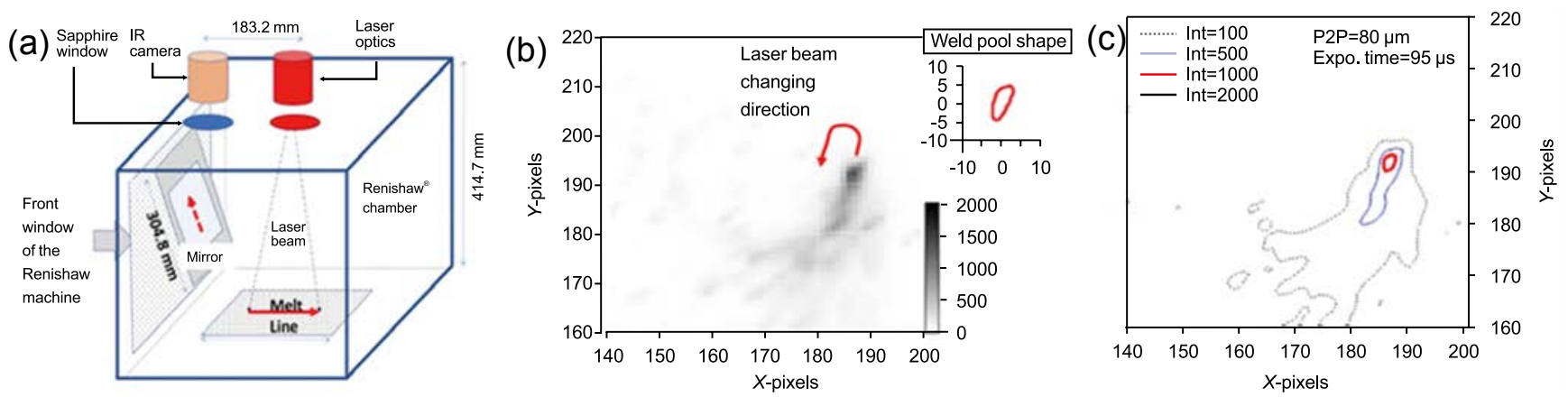

Fig. 11: In situ monitoring of state of melt pool during powder bed fusion process: (a) schematic diagram of paraxial high-speed infrared imaging system ${ }^{[58]}$; (b), (c) infrared image and contour extraction results of melt pool and its surrounding area at a certain time ${ }^{[58]}$ 
Krauss et al. ${ }^{[75]}$ established an in situ monitoring system for SLM process based on infrared thermal imaging. Experiments under different process conditions were conducted and then the infrared images of the melt pool region were collected. The total area, circularity and aspect ratio of the melt pool area were extracted by applying image processing methods. The process parameters were correlated with the infrared image features, and the errors caused by insufficient heat dissipation were studied. The artificial defects in the range of $40 \mu \mathrm{m}-500 \mu \mathrm{m}$ were detected and analyzed. It is found that the total area of the melt pool area can well reflect the fluctuation of the scan length and overlap rate, and the roundness and aspect ratio can be used to detect geometric deviations. The infrared images of the melt pool collected during SLM process were just processed to extract the total area, circularity, and aspect ratio of the melt pool area to reflect the fluctuation of the scan length and overlap rate. The infrared images can also be used as the data set to train a machine learning model to quantitatively predict the fluctuation of the scan length and overlap rate.

Okaro et al. ${ }^{[77]}$ used the coaxial in situ monitoring of melt pool area radiation system to collect a large amount of raw data generated during the SLM process. After testing the mechanical properties of the formed parts, the labels of the corresponding data were obtained. Then, the data set was generated and the principal components were extracted using random singular value decomposition (R-SVD). The semi-supervised learning method was used to model a small number of labeled samples and most unlabeled samples. The success rate of identifying the performance of the formed part is $77 \%$, which demonstrates that the thermal history data collected based on the photodiode is connected to the feasibility of identifying the quality of the parts formed by SLM process. The success rate only being $77 \%$ demonstrates that the semi-supervised learning may not be an industrial friendly strategy for the SLM monitoring. A greater number of labeled samples would improve the success rate. If the method to generate the data set could retain more information of the raw data, the success rate will also be higher.

\subsection{In situ monitoring of scanned layer and powder- spread-layer based on infrared imaging}

As shown in Fig. 12(a), Raplee et al. ${ }^{[28]}$ adopted the detection system based on infrared imaging to develop a method that could accurately correlate the thermal emissivity of the material shown in the collected infrared images with the actual temperature. The thermal history during the process was studied in detail, as shown in Figs. 12(b-e) ${ }^{[28,57]}$. To solve the problem that the accuracy of temperature data was reduced due to the different radiation intensity between the metal powder region and the scanned region of a layer, a method was developed to calibrate the temperature distribution from the infrared image [Fig. 12(e)]. The important thermal feature of the forming process was also determined. After analyzing the infrared imaging data, the transformation process from the metal powder to the scanned region was determined, and the thermal gradient and the advance velocity of solid-liquid interface were approximated, which were correlated with the changes in the internal microstructure of the parts observed in the experiment ${ }^{[57]}$. Because the amount of the images collected
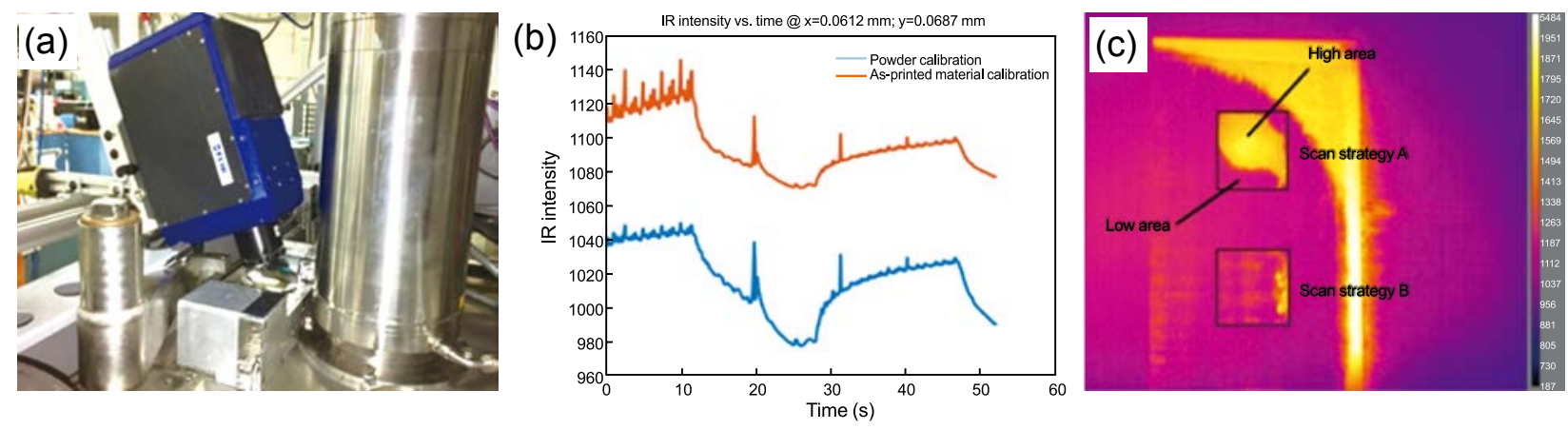

(d)

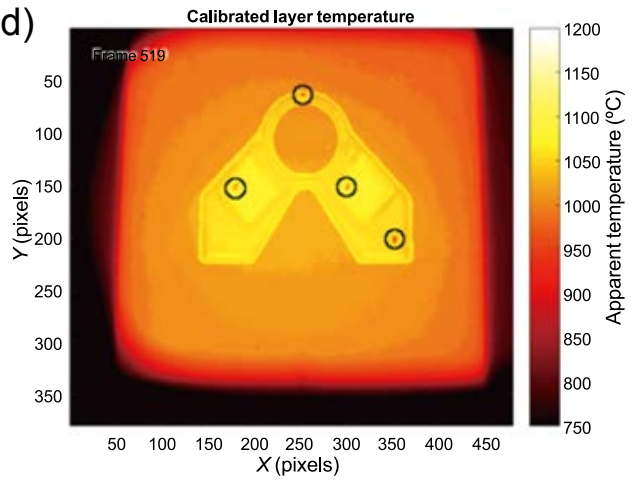

(e)

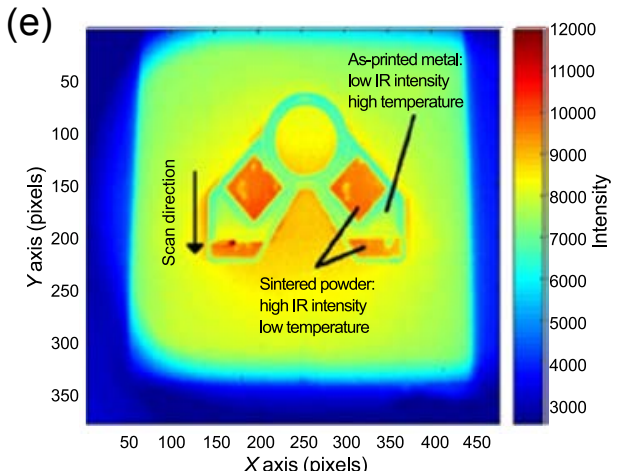

$X$ axis (pixels)

Fig. 12: Paraxial in situ monitoring during PBF manufacturing process based on infrared imaging: (a) schematic diagram of scanned layer monitoring system based on infrared imaging ${ }^{[28]}$, (b) changes of gray value of a position at different infrared images ${ }^{[28]}$, (c) an infrared image of scanned layer ${ }^{[28]}$, (d) and (e) infrared images of scanned layer with temperature difference due to local warping defect ${ }^{[28,57]}$ 
is vast and the labels are easy to get, the task in this research could also be transferred to a pixel value regression task. Deep learning methods could be applied to help accurately correlate the thermal emissivity of the material shown in the collected infrared images with the actual temperature.

As shown in Fig. 13(a), Baumgartl et al. ${ }^{[67]}$ proposed a convolutional neural network that uses the infrared image of the scanned layer collected by the paraxial infrared imaging system as the input to recognize the defects of powder region and spatters in the scanned layer during the SLM process. The structure of the convolutional neural network is shown in Fig. 13(b). To reduce the number of parameters and ensure the performance of $\mathrm{CNN}$, the second and third convolutional layers of the neural network are deep separable convolutions [Fig. 13(c)]. The network obtained $96.80 \%$ accuracy on the collected test set. By calculating the class activation map of a specific input image and its recognition result, the focus of the network during recognition process was explained, and effectiveness of the network was proved. The architecture of CNN proposed in this research is satisfactory. If the $\mathrm{CNN}$ is pre-trained on a data set that possesses more similar images and more effective data augmentation approaches are applied, the accuracy rate will be higher.

Lough et al. ${ }^{[72]}$ used in situ monitoring system based on short wave high speed infrared imaging, as shown in Fig. 14(a), to collect infrared images during the SLM process at a higher frame rate ${ }^{[72]}$. By extracting features such as the time exceeding the threshold, the maximum radiation, and the maximum radiation attenuation rate [Figs. 14 (b), (e)], all the infrared images collected in the same layer were mapped into one image to represent the thermal history of each layer. Then, a three-dimensional reconstruction based on the thermal history of each layer was implemented. Finally, the results of threedimensional reconstruction were compared with the mechanical properties and micro-defects to establish the correlation between local properties and defects. It was found that the thermal characteristics of "exceeding threshold time" had the strongest correlation with the local performance and defects of the part, which provides the basis for the prediction of the quality of the parts during the SLM process ${ }^{[73]}$. The essence of the threedimensional reconstruction of the heat history during SLM is to extract the pixel-level information and then combine the information in the same layer into an image, which is then employed to predict the quality. The efficiency of the method will be improved if the raw images are directly applied to predict the quality instead of being 3D reconstructed.

Williams et al. ${ }^{[76]}$ developed an in situ monitoring system, which integrated the powder bed infrared imaging and melt pool region infrared imaging. The calibration of temperature was carried out through experiments, and the surface temperature of the whole scanned layer, the surface temperature of the powder spread layer and the temperature of the melt pool region were measured. By forming cylindrical parts of different heights, the relationship between interlayer cooling time (ILCT) and the porosity, microstructure and mechanical properties of the parts

(a)

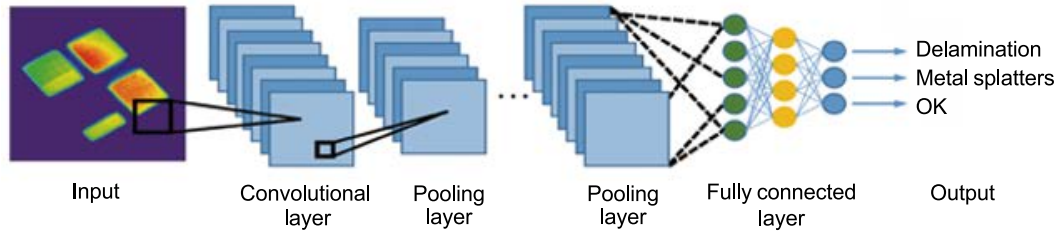

(b)

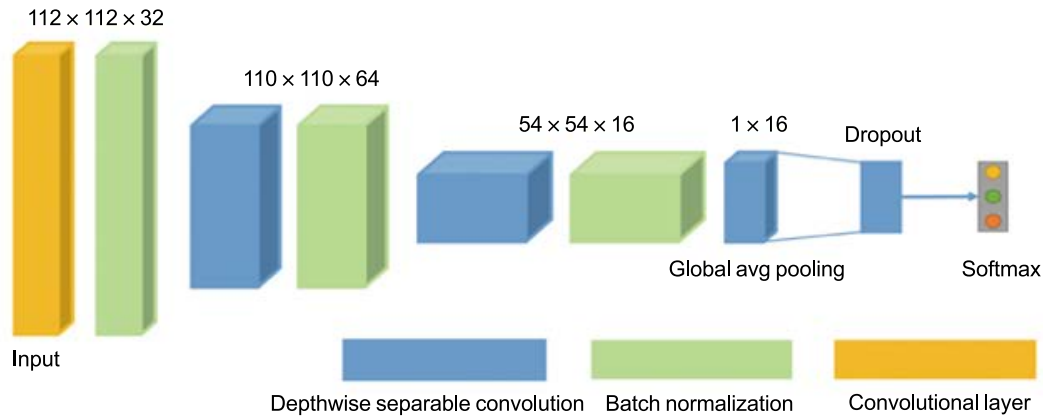

(c)
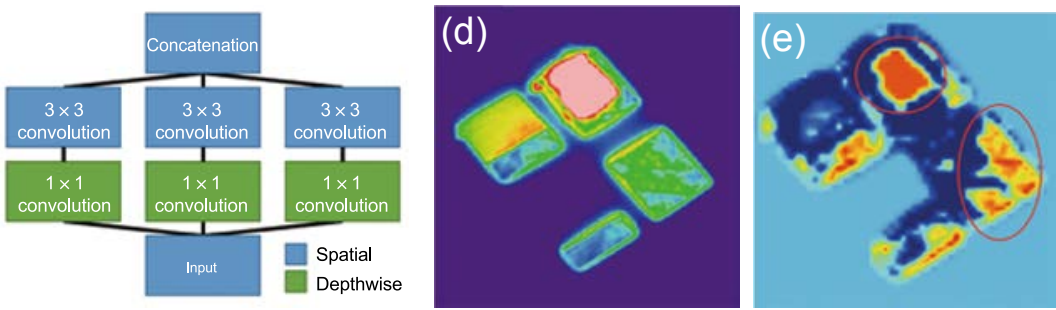

Fig. 13: Defect recognition method of scanned layer images captured by paraxial infrared camera: (a) schematic diagram of defect recognition of infrared images of scanned layer based on CNN ${ }^{[67]}$, (b) structure of CNN ${ }^{[67]}$, (c) schematic diagram of depth separable convolution structure ${ }^{[67]}$, (d) infrared image of a scanned layer ${ }^{[67]}$, (e) gradient-based class activation map ${ }^{[67]}$ 

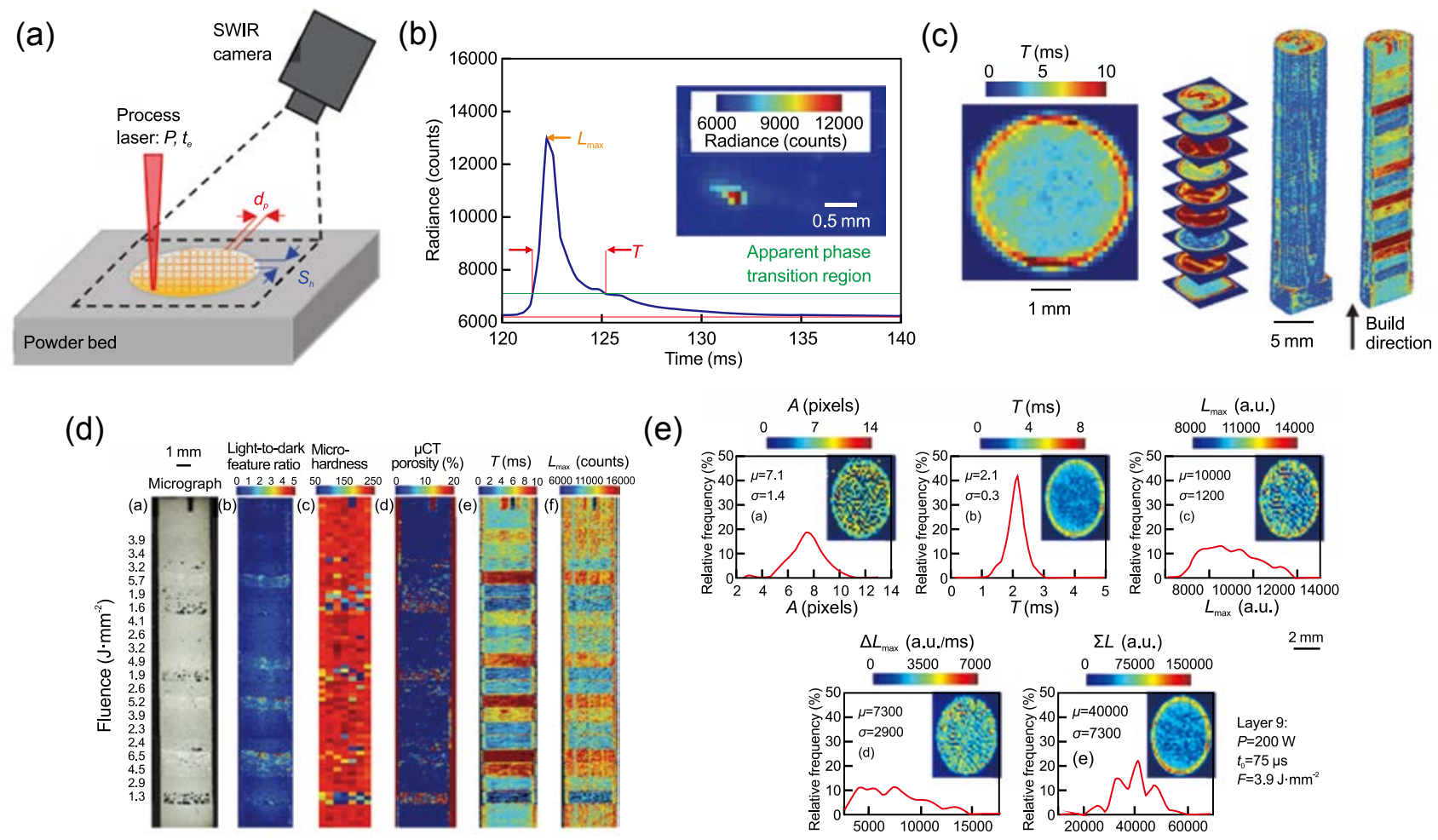

(e)

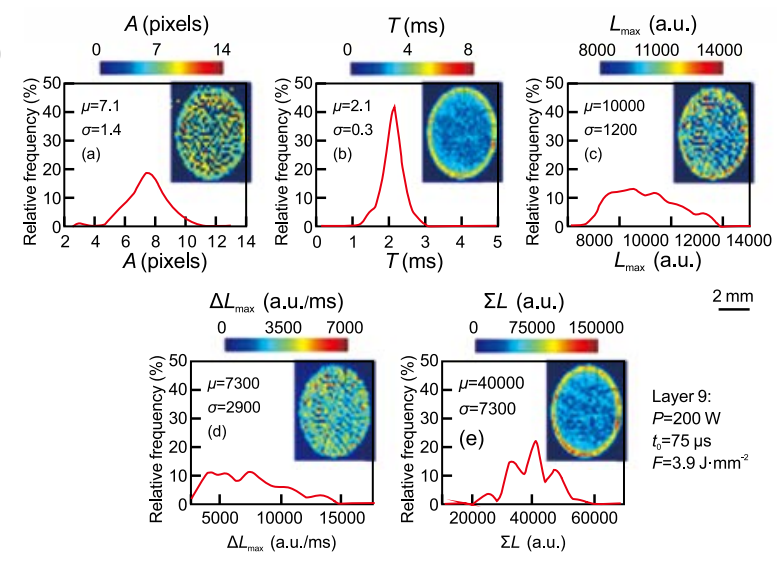

(f)

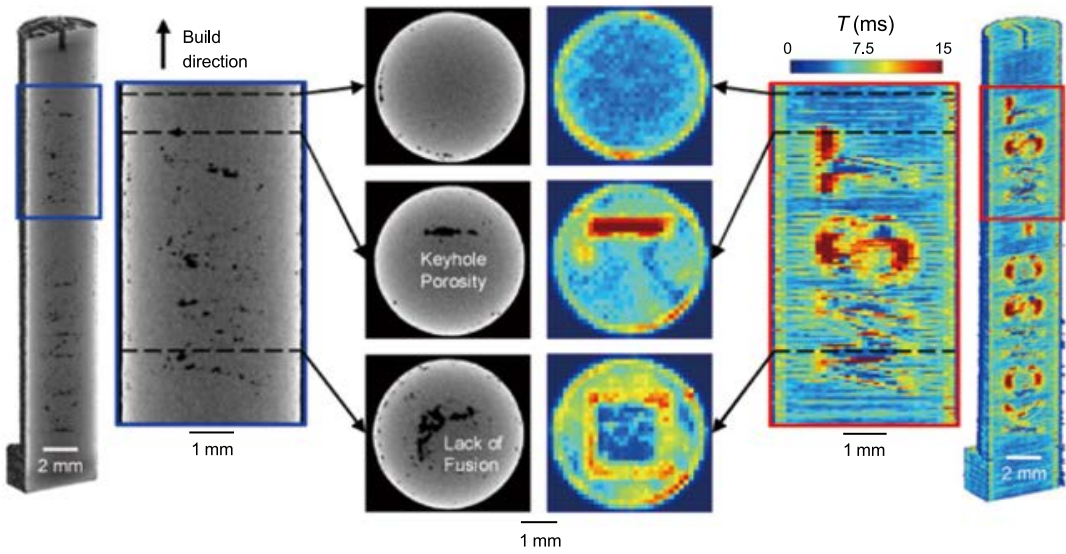

Fig. 14: In situ monitoring of melt pool region during SLM forming process based on infrared image: (a) schematic diagram of high speed short wave infrared imaging system ${ }^{[72,73]}$; (b) changes of pixel value in fixed position of multi-frame infrared images and schematic of threshold value ${ }^{[72]}$; (c) single layer thermal information image and 3D reconstruction results obtained by mapping method of "super threshold time" ${ }^{[72]}$; (d) comparison between actual defect detection results and 3D reconstruction results based on infrared thermal information ${ }^{[72]}$; (e) examples of five thermal characteristics, such as single-layer molten pool area, time over threshold, maximum radiation and maximum radiation attenuation rate ${ }^{[73]}$; (f) comparison between actual defect detection results and three-dimensional reconstruction results obtained by mapping method of "super threshold time" [73]

was studied. It is found that the surface temperature of scanned layer and the temperature of powder spread layer are important factors affecting the quality of parts during SLM process. A different ILCT leads to a different shape of melt pool. Shorter ILCT makes melt pool a stable shape and could eliminate the hole defects to a certain extent. A quantitative model, which could be generated by data-driven machine learning methods, may make this approach more accurate. The relationship between ILCT and the porosity, microstructure and mechanical properties of the parts may be modeled by regression models, such as DNN and SVR.

\subsection{In situ monitoring of scanned layer and powder spread layer based on visible light imaging}

Foster et al. ${ }^{[18]}$ used the high resolution imaging system shown in Figs. 15(a) and (b) to collect and analyze the images of the powder spread layer and scanned layer [Figs. 15(c)-(e)]. The quality of each scanned layer and powder spread layer during SLM process was identified and the 3D reconstruction of the testing results was realized. Abdelrahman et al. ${ }^{[19]}$ also used the slice result of the $3 \mathrm{D}$ model of the part as a mask to associate it with the images of the corresponding layer, and then 

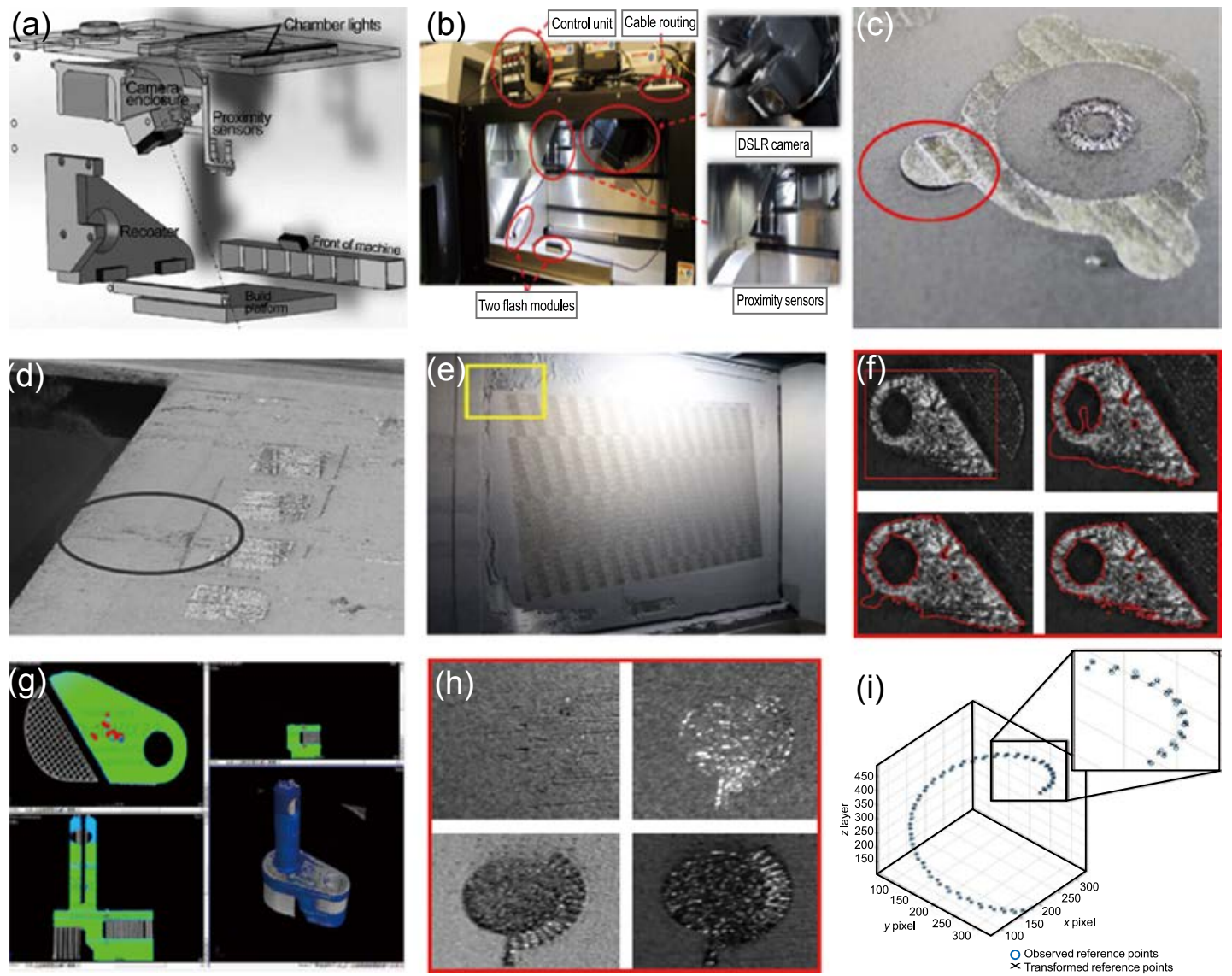

Fig. 15: Paraxial in situ monitoring of scanned layer during SLM process: (a and b) physical diagram and schematic diagram of in situ monitoring system ${ }^{[19,22]},(\mathbf{c}, \mathbf{d}$ and $\mathrm{e})$ defects in scanned layer and powder spread layer ${ }^{[18]}$, ( $f$ and $g$ ) scanned layer image segmentation and results of three dimensional reconstruction ${ }^{[19]}$, $(h)$ image of scanned layer and powder spread layer ${ }^{[22]}$, (i) defect recognition results ${ }^{[22]}$

implemented the segmentation of the part region in the images of each layer. By applying the level set image segmentation algorithm to the image of the segmented parts region, automatic detection of the defects of scanned layer was realized. Finally, $3 \mathrm{D}$ reconstruction was carried out based on the detection results of each layer [Figs. 15(f), (g)]. Reutzel et al. ${ }^{[22]}$ also used affine transformation to convert the high resolution 3D CT detection results of the formed parts into the spatial positions of the acquired images, which were employed as labels. After feature extraction of the collected images, support vector machines were trained and generated recognition accuracy above $80 \%$. The recognition results are shown in Fig. 15(i). The image segmentation approach applied in the research is based on the level set, which is an interactive image segmentation algorithm and takes additional initial contour as input besides the original image. The initial contour may directly affect the final segmentation result. The mass image data could be applied as the data set for a deep learning based image segmentation approach, which does not need initial contour, takes the original image as input and directly generates accurate segmentation results.

As shown in Fig. 16(a), Grasso et al. ${ }^{[23]}$ used the paraxial in situ monitoring system for EBM process. A method for detecting the uniformity of the powder spread layer based on high-resolution imaging combined with image segmentation and morphological operations was proposed. In addition, a method for detecting the local overheating of scanned layer based on high-speed imaging combined with difference of pixels between frames was also proposed. The defects in the powder spread layer and scanned layer can be quickly detected by the above two methods, as shown in Figs. 16(b) and (c). As shown in Figs. 16(d) and (e), Grasso et al. ${ }^{[24]}$ also independently developed the experimental device. The imaging system was equipped with six directional light sources consisting of LED strip lights placed in six different locations on the ceiling of the build chamber, which enhanced the quality of the captured images. The image segmentation methods based on different active contours under different illumination conditions were studied. It is found that under appropriate lighting conditions, the geometric deviation detection can be accomplished with a lower error by combining the image preprocessing algorithm with the image segmentation algorithm. As shown in Fig. 16(g), Grasso et al. ${ }^{[25]}$ applied the paraxial in situ monitoring system to SLM process, and proposed a statistical process monitoring method for processing images with complex geometric shapes. By coupling the edge-based and region-based image segmentation algorithms, the contours of the scanned layer were reconstructed and compared with the standard geometry of the scanned layer. Finally, the defects of each layer were detected successfully during the SLM process. A deep learning based image segmentation method, which fuses the 

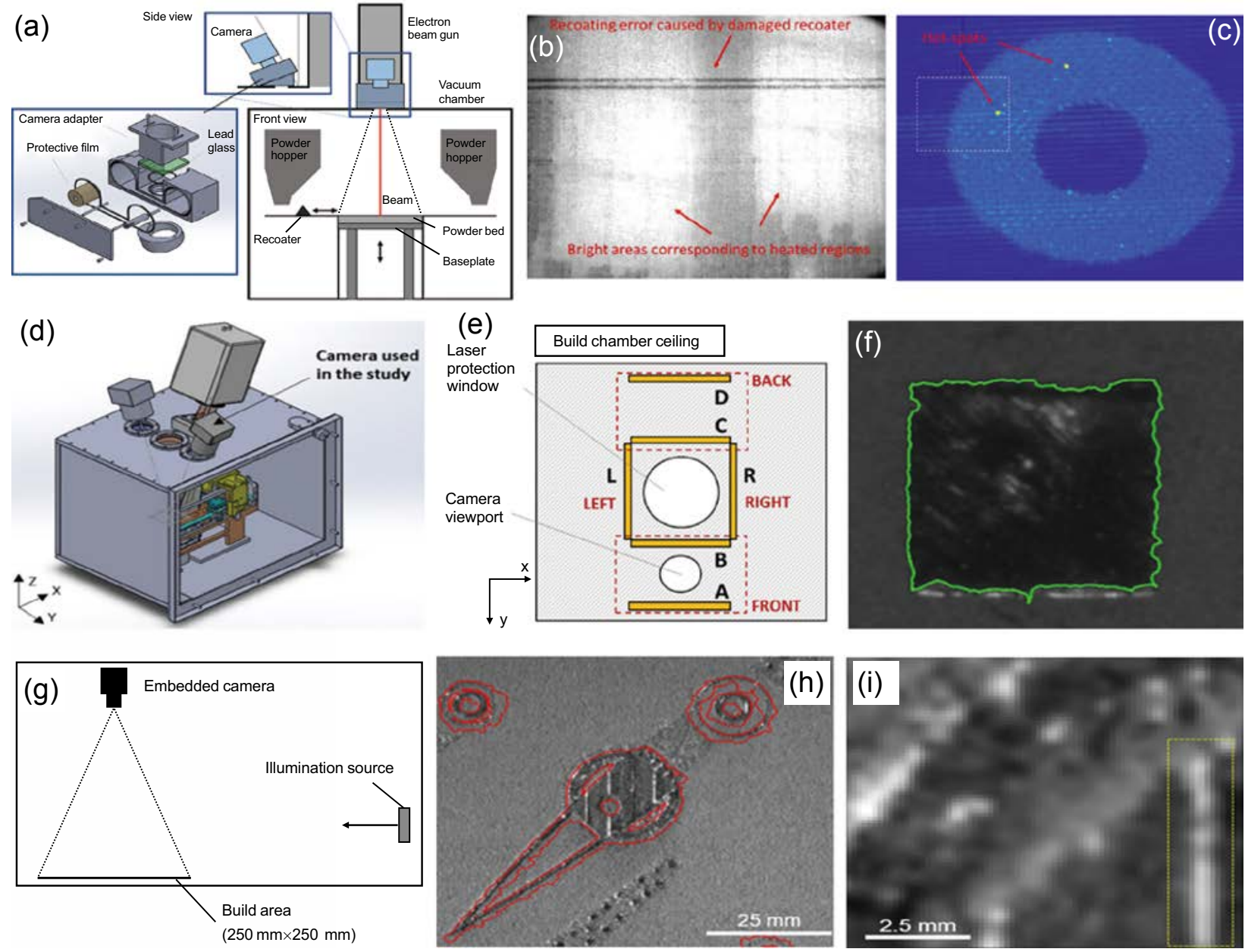

Fig. 16: Paraxial in situ monitoring of powder spread layer and scanned layer during PBF process: (a) schematic diagram of in situ monitoring during EBM process ${ }^{[23]}$, (b) scratch defects on powder spread layer ${ }^{[23]}$, (c) local overheating defect on scanned layer ${ }^{[23]}$, (d) self-developed SLM equipment with visible light camera ${ }^{[24]}$, (e) schematic diagram of illumination scheme ${ }^{[24]}$, (f) contour in a scanned layer extracted by edgeless active contour algorithm ${ }^{[24]}$ ( $(\mathrm{g})$ schematic diagram of EOS built-in paraxial in situ monitoring system ${ }^{[25]}$, (h) and (i) detection results of contour edge of scanned layer and defects in scanned layer ${ }^{[25]}$

low-level information of image elements and high-level semantic information, could make full use of the massive image data and detect small defects during the SLM process more accurately than classical image segmentation methods.

Furumoto et al. ${ }^{[46]}$ observed the solidification process of iron-based powder melts by using the high-speed imaging system, and studied the influence of thickness of layer on the solidification characteristics of the melt, which directly reflected the formation process of the melt under the action of laser. The surface tension of the metal liquid and the bonding force caused by the wetting characteristics of the metal liquid on the front layer will affect the morphology of the melt ${ }^{[48]}$. Furumoto et al. ${ }^{[48]}$ also observed the melt pool and its solidification process during the formation of a single track, and found that the size of the melt pool increased with the increase of laser power and decreased with the increase of scanning speed. The area of melt pool is usually larger than the size of laser beam spot. As the layer thickness increases, the formation of melt pool becomes unstable or even chaotic. After solidification, an uneven molten track would be formed, and powder spatters would be more obvious. The increased layer thickness also causes the molten powder to form a ball, which is difficult to be wet with the substrate. The results of this research have important theoretical guiding significance. When the research was completed, the efficient artificial intelligence algorithms, such as AlexNet and EfficientNet, have not been proposed because of the hardware restriction. If the collected image data are fully mined and the quantitative model is built by machine learning methods or deep learning methods, the data will have greater value.

Kleszczynski et al. ${ }^{[47]}$ proposed two methods to analyze the quality of scanned layer and powder spread layer during SLM process. One method is based on the imaging system of the high-resolution visible light camera, as shown in Figs. 17(a) and (b) ${ }^{[47]}$. An image-based bulges detection algorithm of the scanned layer was developed, and a quantitative measurement method for analyzing the parts regions of each forming layer was proposed ${ }^{[50]}$. As shown in Figs. 17(c)-(f), obvious forming layer defects, such as scanned layer bulges, were detected on the microscopic scale, and the geometric features of the defects in 

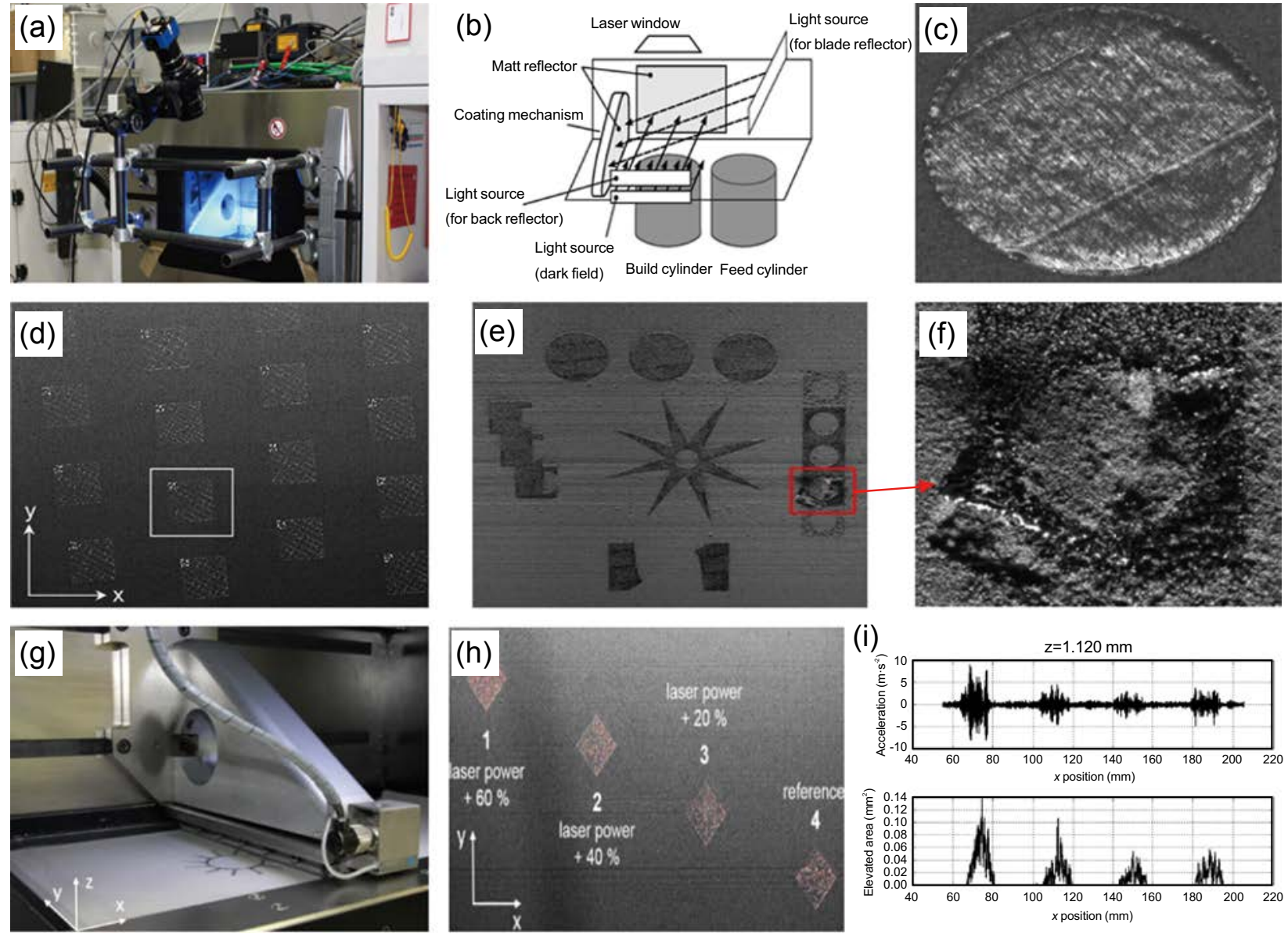

Fig. 17: Paraxial in situ monitoring system of powder spread layer and scanned layer during SLM process: (a) and (b) physical diagram and schematic diagram of system based on high resolution imaging ${ }^{[47]}$, (c) bulges in scanned layer ${ }^{[47]}$, (d) bulge in powder spread layer ${ }^{[50]}$, (e ) and (f) collapse of scanned area due to weak support ${ }^{[51]}$, (g) recoater equipped with acceleration sensor ${ }^{[52]}$, (h) and (i) scanned layer with bulges and acceleration measurement results ${ }^{[52]}$

the image were also measured to realize the positioning of the key areas ${ }^{[51]}$. Another method is to combine the image of the scanned layer collected by the high-resolution imaging system, and use the acceleration sensor as shown in Fig. 17(g) to detect the collision of the recoater and the bulges in the scanned layer. Thereby, the threshold of detection of bulges in the scanned layer based on images was set ${ }^{[52]}$. Finally, the knowledge base for the detection of the bulges in the scanned layer during the SLM process was established. This research proposed a method of quality monitoring by processing the signals from multi-type sensors and provided a new idea for monitoring the quality of scanned layer. With the improvement of measurement accuracy of various sensors and the development of big data processing algorithms, the fusion of the signals collected from multi-type sensors will be one of the main directions in future research.

As shown in Fig. 18(a), Scime et al. ${ }^{[62]}$ used the EOS M290 built-in paraxial camera to collect the images of the powder spread layer with defects [Figs. 18(b), (c) and (d)]. The subimages of defects shown in Fig. 18(e) were obtained by manual interception of the defect areas in the images. With 37 convolution kernels as pattern extractors and convolution results of each pixel of the original image as basic data sets, 100 basic visual words were generated by clustering algorithm
[Fig. 18(f)]. Then, the data set for KNN (K-nearest neighbours) inferencing was acquired. During detection, the original highresolution image was meshed, and the visual word histogram of the sub-image was employed as the input of KNN to obtain the recognition result of the sub-image [Fig. 18(g)]. As shown in Fig. 18(h), to improve the performance of defect recognition, Scime et al. ${ }^{[63]}$ proposed the convolutional neural network using multi-scale gray image as input, and trained the network through transfer learning method to overcome the obstacle of small data scale. The confusion matrix representing the classification performance of the neural network is shown in Fig. 18(i). The defect detection result of an image of powder spread layer in Fig. 18(c) is shown in Fig. 18(j). The quality monitoring task during SLM process was converted to an object detection task. KNN and CNN were applied to recognize the sub-images of the original images. Sliding window, which is essentially an enumeration method, is used as the detection method, which is time-consuming. Many real time object detection approaches with high mAP (mean average precision) should be able to solve this problem in a better way.

As shown in Figs. 19(a) and (b), Lu et al. ${ }^{[70]}$ built a set of in situ monitoring devices for SLM process. Light emitting diode (LED) light strips were installed onto the chamber walls of the 

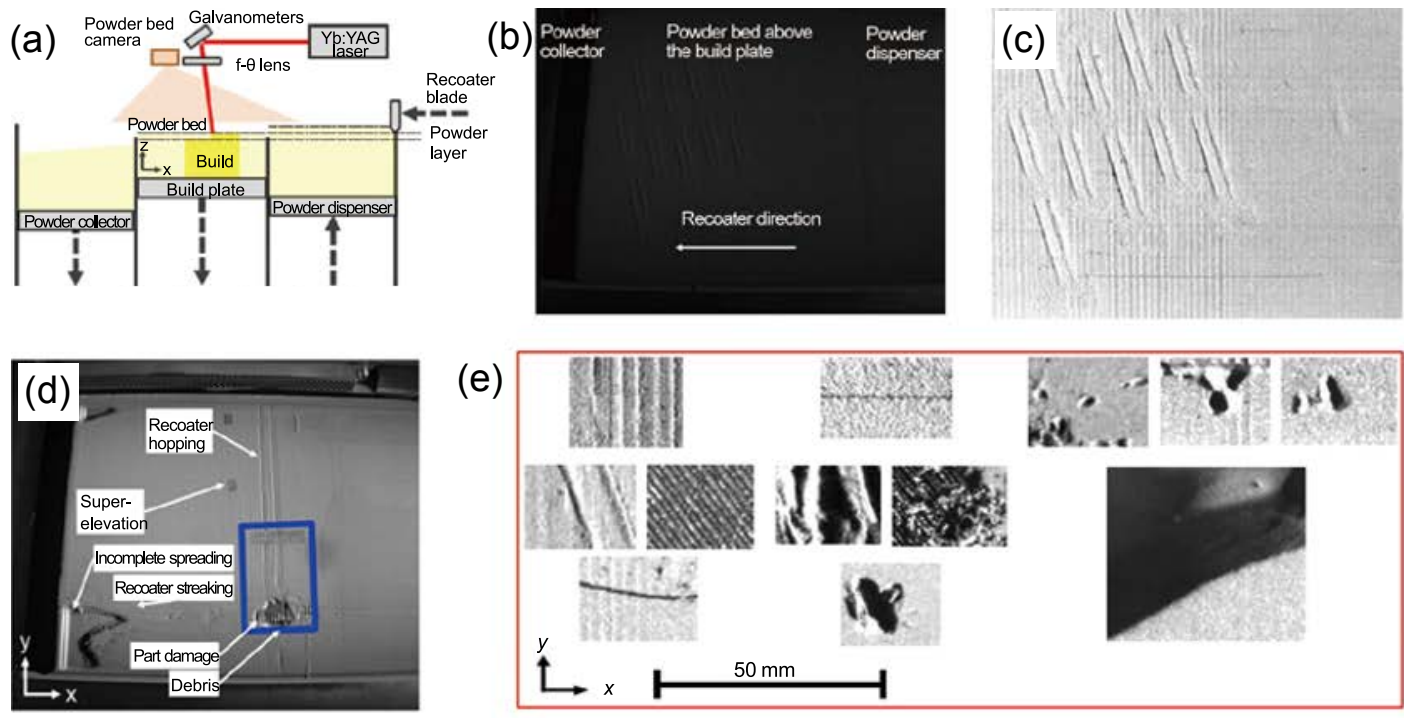

(e)
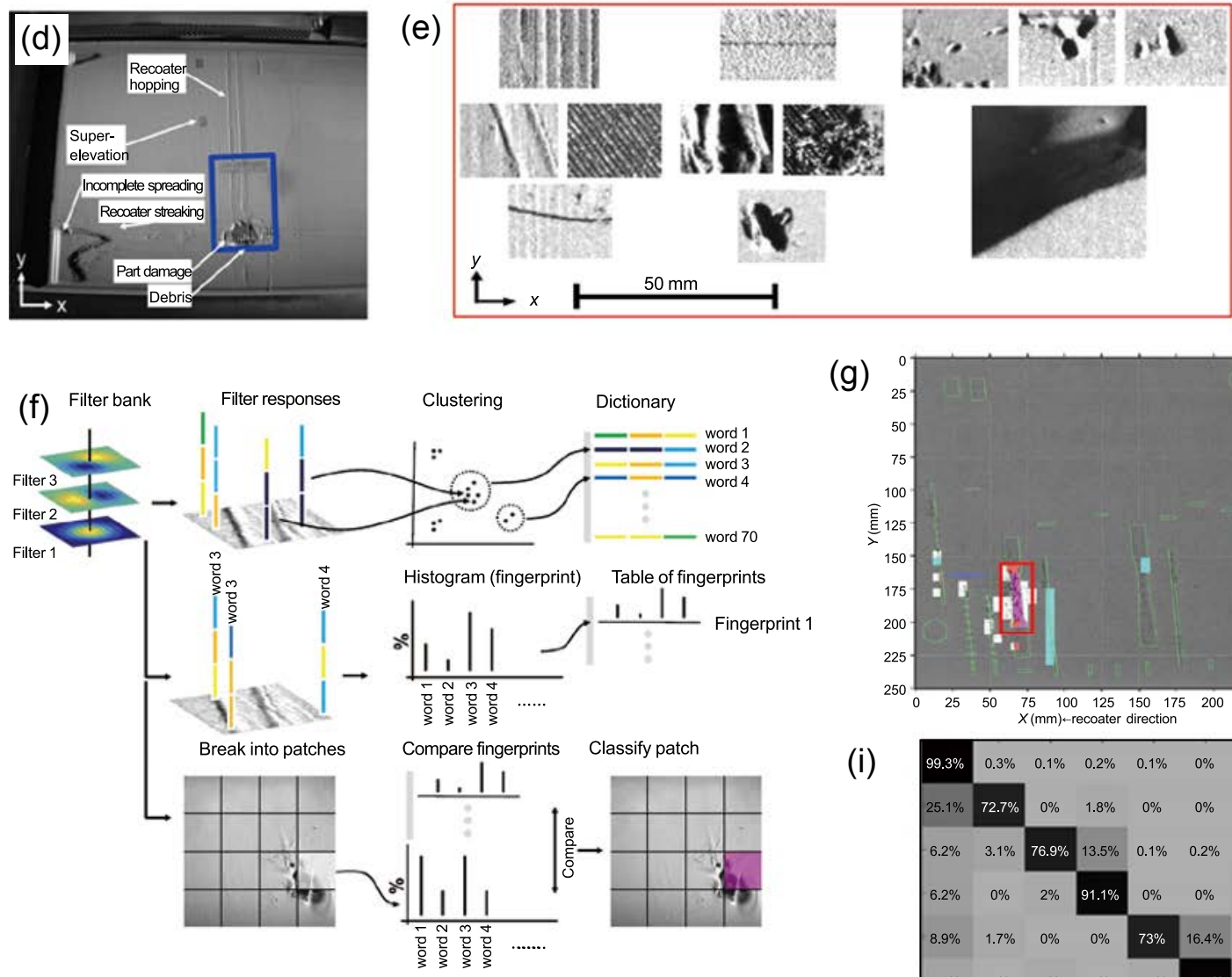

(g)

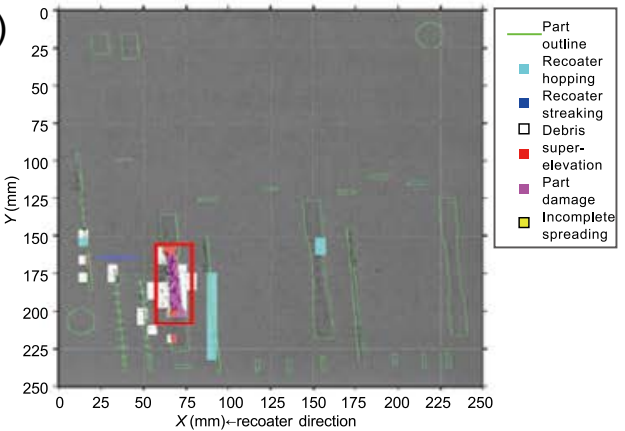

(i)
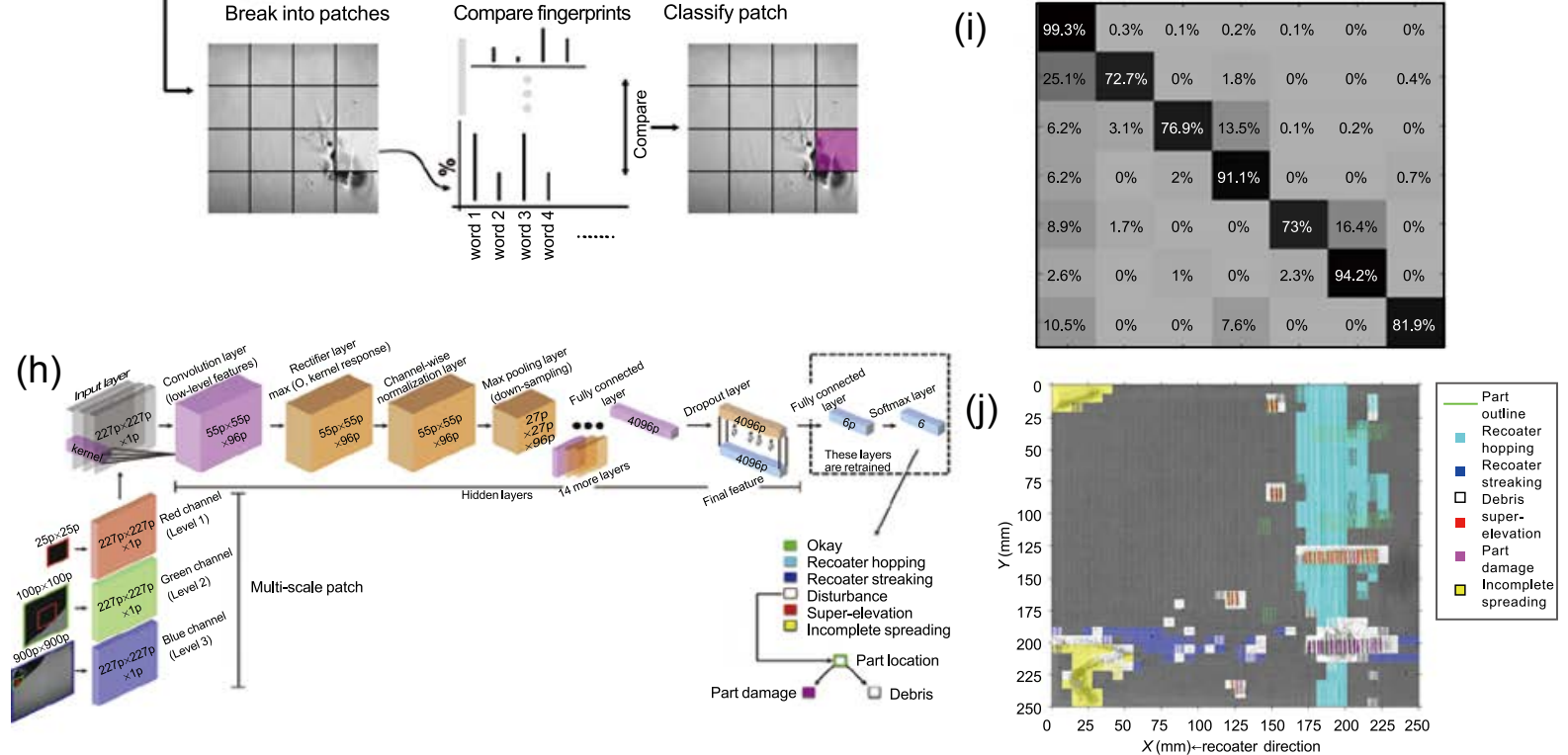

Fig. 18: Schematic diagram of paraxial image acquisition system built in EOS M290 ${ }^{[62]}$ (a), original image captured ${ }^{[62]}(b)$, result of brightness enhancement of captured image ${ }^{[62]}$ (c), image of powder spreading layer with defects ${ }^{[63]}$ (d),examples of six types of defects in powder spread layer ${ }^{\left[{ }^{[62,63]}\right.}(\mathrm{e})$, schematic diagram of defect classification algorithm based on visual word bag and $\mathrm{KNN}^{[62]}(\mathrm{f})$, example of defect detection results in scanned layer image ${ }^{[62]}(\mathrm{g})$, multi-scale CNN structure for defect classification ${ }^{[63]}(\mathrm{h})$, confusion matrix representing the performance of MsCNN ${ }^{[63]}$ (i), example of defect detection results in powder spread layer image ${ }^{[63]}(j)$

machine to achieve uniform lighting during image capturing. The Nikon D500 DSLR camera was used to capture optical images during the printing process ${ }^{[70]}$. Ten samples were formed under different process parameters by changing laser power and scanning speed, and the images of powder spread layer and scanned layer during the process were collected, as shown in Figs. 19(c)-(e) and (h). The image processing technology for images of both scanned layer and powder spread layer was developed, and features were quantified and recognized. The experimental results demonstrated that the test results of density 

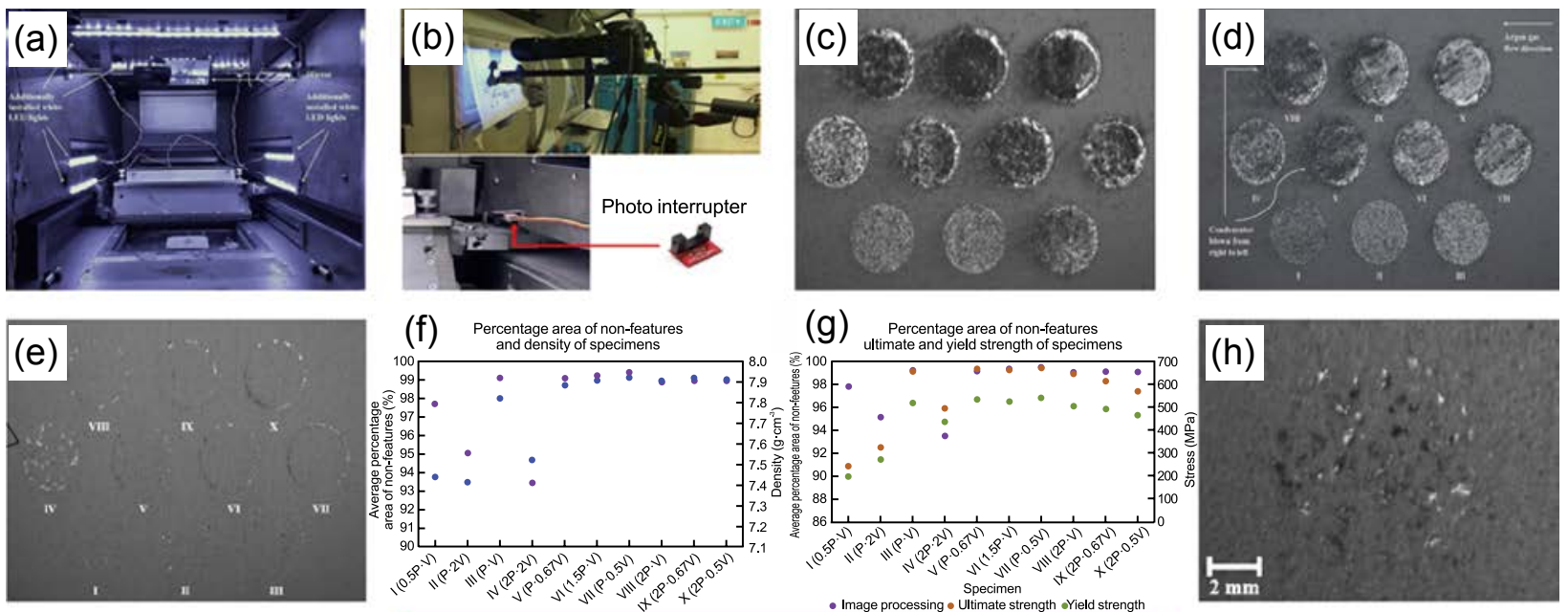

(g)
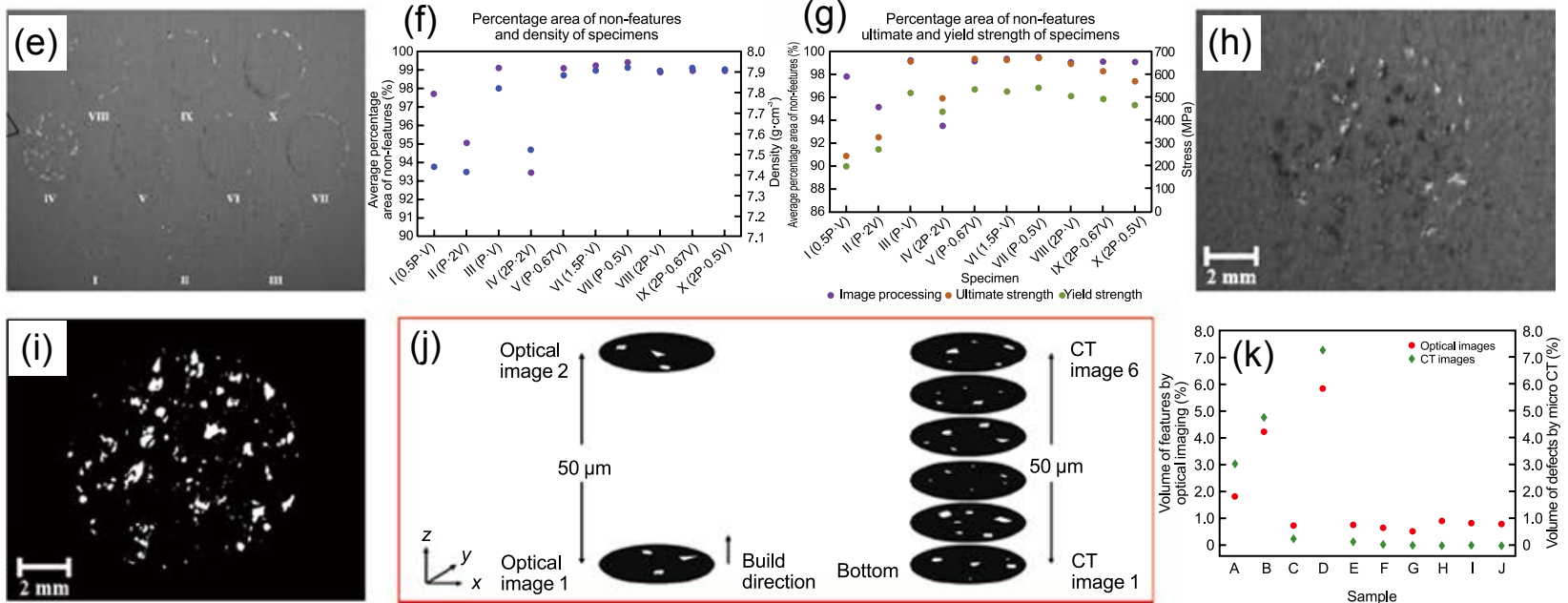

Fig. 19: Paraxial in situ monitoring of scanned layer and powder spread layup-forming layer during SLM process: (a) illumination layout designed for in situ monitoring during SLM process ${ }^{[70]}$; (b) schematic diagram of image acquisition system ${ }^{[70]}$; (c) image of scanned layer with defects ${ }^{[70]}$; (d) image of scanned layer with black slag ${ }^{[70]}$; (e) image of uneven spread powder layer ${ }^{[00]}$; (f) relationship between results of image quantitative analysis and density ${ }^{[70]}$; $(g)$ relationship between results of image quantitative analysis and mechanical properties ${ }^{[70] \text {; }}$ ( $\mathrm{h}$ and i) image collected during SLM process and its binary processing results ${ }^{[71]}$; (j) number of optical images and CT images collected under condition of single layer thickness distance ${ }^{[71]}$; $(k)$ relationship between image quantitative analysis results of each sample process and results of nondestructive testing ${ }^{[71]}$

based on Archimedes' principle and mechanical properties of sample were correlated with the quantitative results of the image of sample during SLM process [Figs. 19 (f), (g)], which proved that the in situ monitoring system based on image processing was feasible to infer the mechanical properties of parts. Lu et al. ${ }^{[71]}$ conducted nondestructive testing on the parts by Micro CT based on the previous study ${ }^{[70]}$, and reconstructed the CT scan results of the components. The correlation between quantified features of images collected during SLM process and actual defects was verified. The feasibility of using the image of powder spread layer and scanned layer during SLM process to evaluate the defects of parts was also proved. If statistical models or machine learning models, which could predict the mechanical properties and the defects of parts quantitatively, could be trained based on the images collected during the SLM process, the approaches proposed in the above research will be more useful in engineering applications.

\subsection{Summary of image analysis methods used in the in situ monitoring for SLM process}

Table 2 summarizes the image analysis methods applied in SLM process. According to the semantic level of the image

Table 2: Summary of image analysis methods used in the in situ monitoring for SLM process

\begin{tabular}{|c|c|c|c|}
\hline \multirow{2}{*}{ Brief description } & \multicolumn{3}{|c|}{ Semantic level of image analysis } \\
\hline & Low & Middle & High \\
\hline Typical algorithms & Image processing & $\begin{array}{l}\text { Image processing + } \\
\text { machine learning }\end{array}$ & Deep learning \\
\hline \multirow[t]{2}{*}{ Comments } & $\begin{array}{l}\text { Extract the basic features of } \\
\text { images manually, such as } \\
\text { brightness, geometry and texture }\end{array}$ & $\begin{array}{c}\text { Extract the basic features of } \\
\text { images manually and recognize } \\
\text { the defects automatically }\end{array}$ & $\begin{array}{l}\text { Extract the basic features of } \\
\text { images automatically and } \\
\text { recognize the defects } \\
\text { automatically }\end{array}$ \\
\hline & Qualitative analysis & Quantitative analysis & $\begin{array}{l}\text { Automatic, accurate and } \\
\text { quantitative analysis }\end{array}$ \\
\hline References & $\begin{array}{c}{[9,12-19,23-25,27-28,46-49} \\
51-52,57-59,70-73,75-76]\end{array}$ & $\begin{array}{c}{[22,30,32,35-39,60-62,64} \\
66-67]\end{array}$ & {$[11,29,31,63,67]$} \\
\hline
\end{tabular}


information processed, the image analysis methods are divided into three categories: low, middle, and high semantic level image analysis methods. It can be seen from Table 2 that most of the existing image analysis methods of in situ monitoring during SLM process are mainly based on traditional image processing approaches and traditional machine learning models. The low semantic level image analysis methods and middle semantic level image analysis methods are low in efficiency and accuracy compared with the high semantic level image analysis methods. The high semantic level image analysis methods based on intelligent deep neural networks have already been used for in situ monitoring for SLM process and shown satisfactory results.

\section{Summary}

The in situ monitoring methods during selective laser melting process based on images are reviewed. Firstly, the significance of in situ monitoring during SLM process was introduced. Then, the image information source of SLM process, the image acquisition systems for different detection objects (the molten pool region, the scanned layer and the powder spread layer) and the methods of the image information analysis, detection and recognition are reviewed and analyzed. It is found that the existing image analysis methods of in situ monitoring during SLM process are mainly based on traditional image processing approaches and traditional machine learning models. The in situ monitoring during SLM process based on images has been proved to be an effective means of monitoring the process stability and quality. It is believed that the in situ monitoring during SLM process based on images will have the following development trends:

(1) Intelligent perception and inferencing

With the rapid development of image-based computer vision algorithms, the intelligent deep neural networks that perform well in natural image detection and segmentation tasks will be used in the in situ monitoring during SLM process, which greatly improves the efficiency and accuracy of online detection, and liberates researchers from the complicated and inefficient feature design work.

(2) Multi-sources and multi-scales image information fusion analysis

In addition to the image acquisition systems for visible and infrared bands, other nondestructive testing systems (such as $\mathrm{X}$-ray microimager, etc.) also tend to be used for the in situ monitoring during SLM process, which could generate multisources and multi-scales image information. Therefore, in situ monitoring during SLM process will be implemented in a more comprehensive and detailed way through the fusion and analysis of multi-sources and multi-scales image information.

(3) Industrialization

The high-end industry's continuous pursuit of component quality stability and the achievement of in situ monitoring during SLM process based on a more intelligent, efficient, comprehensive, and detailed way will definitely promote the implementation and industrialization of this technology.

(4) Establishment of technical standards and multi-terminal data sharing

Manufacturing execution system (MES), Internet of Things and 5G technology will enable the interconnection of many in situ monitoring systems for SLM process and render the image database for in situ monitoring more complete. In addition, the industrialization of this technology will inevitably give birth to the establishment of this technical standard, which will ultimately help the in situ monitoring systems of different terminals to achieve data sharing and improve the monitoring performance, so as to provide high-quality decision-making information for the next step of feedback control research.

\section{Acknowledgements}

This work is financially supported by the KGW Program (Grant No. 2019XXX.XX4007Tm) and the National Natural Science Foundation of China (Grant Nos. 51905188, 52090042 and 51775205).

\section{References}

[1] Yap C Y, Chua C K, Dong Z L, et al. Review of selective laser melting: Materials and applications. Applied Physics Reviews, 2015, 2(4): 041101. doi.org/10.1063/1.4935926

[2] Wei Q S, Song B, Wen S F, et al. Metal powder bed laser additive manufacturing technology. Beijing: Chemical Industrial Press, 2019: 2-25. (In Chinese)

[3] Yang L L. Study on spatter behavior and molten pool characteristics in selective laser melting based on highspeed photography technique. Master Dissertation, Huazhong University of Science and Technology, 2019. (In Chinese)

[4] Lott P, Schleifenbaum H, Meiners W, et al. Design of an optical system for the in situ process monitoring of selective laser melting (SLM). Physics Procedia A, 2011, 12: 683-690.

[5] Everton S K, Hirsch M, Stravroulakis P, et al. Review of in-situ process monitoring and in-situ metrology for metal additive manufacturing. Materials \& Design, 2016, 95: 431-445.

[6] Grasso M, Colosimo B M. Process defects and in situ monitoring methods in metal powder bed fusion: A review. Measurement Science and Technology, 2017, 28(4): 044005.

[7] Ding H Y, Wu S T, Yuan K, et al. Quality control of precise metal parts produced by selective laser melting. Hot Working Technology, 2020, 49(22): 12-15. (In Chinese)

[8] Yeung H, Yang Z, Yan L. A meltpool prediction based scan strategy for powder bed fusion additive manufacturing. Additive Manufacturing, 2020, 35: 101383.

[9] Yang D K, Li H, Liu S, et al. In situ capture of spatter signature of SLM process using maximum entropy double threshold image processing method based on genetic algorithm. Optics \& Laser Technology, 2020, 131: 106371.

[10] Gould B, Wolff S, Parab N, et al. In situ analysis of laser powder bed fusion using simultaneous high-speed infrared and x-ray imaging. JOM, 2021, 73(1): 201-211.

[11] Elwarfalli $H$, Papazoglou D, Erdahl D, et al. In situ process monitoring for laser-powder bed fusion using convolutional neural networks and infrared tomography. In: 2019 IEEE National Aerospace and Electronics Conference (NAECON), IEEE, 2019: 323-327. 
[12] Yakout M, Phillips I, Elbestawi M A, et al. In-situ monitoring and detection of spatter agglomeration and delamination during laser-based powder bed fusion of Invar 36. Optics \& Laser Technology, 2020: 106741.

[13] Yang Lihang, Lo Lydia, Ding Sijie, et al. Monitoring and detection of meltpool and spatter regions in laser powder bed fusion of super alloy Inconel 625. Progress in Additive Manufacturing, 2020, 5(4): 367-378.

[14] Wang D, Wu S B, Fu F, et al. Mechanisms and characteristics of spatter generation in SLM processing and its effect on the properties. Materials \& Design, 2017, 117: 121-130.

[15] Craeghs T, Bechmann F, Berumen S, et al. Feedback control of layerwise laser melting using optical sensors. Physics Procedia, 2010, 5: 505-514.

[16] Craeghs T, Clijsters S, Yasa E, et al. Online quality control of selective laser melting. In: Proceedings of the 20th Solid Freeform Fabrication (SFF) Symposium, Austin (Texas), 8-10 August, 2011: 212-226.

[17] Craeghs T, Clijsters S, Kruth J P, et al. Detection of process failures in layerwise laser melting with optical process monitoring. Physics Procedia, 2012, 39: 753-759.

[18] Foster B, Reutzel E, Nassar A, et al. Optical, layerwise monitoring of powder bed fusion. In: Proc. Solid Freeform Fabrication Symposium, Austin, TX, Aug. 2015: 10-12.

[19] Abdelrahman M, Reutzel E W, Nassar A R, et al. Flaw detection in powder bed fusion using optical imaging. Additive Manufacturing, 2017, 15: 1-11.

[20] Liu $Y$, Blunt L, Zhang $Z \mathrm{H}$, et al. In-situ areal inspection of powder bed for electron beam fusion system based on fringe projection profilometry. Additive Manufacturing, 2020, 31: 100940.

[21] Bartlett J L, Heim F M, Murty Y V, et al. In situ defect detection in selective laser melting via full-field infrared thermography. Additive Manufacturing, 2018, 24: 595-605.

[22] Gobert C, Reutzel E W, Petrich J, et al. Application of supervised machine learning for defect detection during metallic powder bed fusion additive manufacturing using high resolution imaging. Additive Manufacturing, 2018, 21: 517528.

[23] Grasso M, Valsecchi G, Colosimo B M. Powder bed irregularity and hot-spot detection in electron beam melting by means of in-situ video imaging. Manufacturing Letters, 2020, 24: 47-51.

[24] Caltanissetta F, Grasso M, Petrò S, et al. Characterization of insitu measurements based on layerwise imaging in laser powder bed fusion. Additive Manufacturing, 2018, 24: 183-199.

[25] Pagani L, Grasso M, Scott P J, et al. Automated layerwise detection of geometrical distortions in laser powder bed fusion. Additive Manufacturing, 2020, 36: 101435.

[26] Croset G, Martin G, Josserond C, et al. In-situ layerwise monitoring of electron beam powder bed fusion using nearinfrared imaging. Additive Manufacturing, 2021, 38: 101767.

[27] Craeghs T, Clijsters S, Yasa E, et al. Determination of geometrical factors in layerwise laser melting using optical process monitoring. Optics and Lasers in Engineering, 2011, 49(12): 1440-1446.

[28] Raplee J, Plotkowski A, Kirka M M, et al. Understanding the thermal sciences in the electron beam melting process through in-situ process monitoring. In: Proc. Nondestructive Characterization and Monitoring of Advanced Materials, Aerospace, and Civil Infrastructure 2017. International Society for Optics and Photonics, 2017, 10169: 1016900.

[29] Ye Dongsen, Hsi Fuh Jerry Ying, Zhang Yingjie, et al. In situ monitoring of selective laser melting using plume and spatter signatures by deep belief networks. ISA Transactions, 2018, 81: $96-104$
[30] Ye Dongsen, Zhu Kunpeng, Fuh Jerry Ying Hsi, et al. The investigation of plume and spatter signatures on melted states in selective laser melting. Optics \& Laser Technology, 2019, 111: 395-406.

[31] Zhang Yingjie, Hong Geok Soon, Ye Dongsen, et al. Extraction and evaluation of melt pool, plume and spatter information for powder-bed fusion AM process monitoring. Materials \& Design, 2018, 156: 458-469.

[32] Zhang Yingjie, Fuh Jerry Y H, Ye Dongsen, et al. In-situ monitoring of laser-based PBF via off-axis vision and image processing approaches. Additive Manufacturing, 2019, 25: 263-274.

[33] Ye Dongsen, Hong Geok Soon, Zhang Yingjie, et al. Defect detection in selective laser melting technology by acoustic signals with deep belief networks. The International Journal of Advanced Manufacturing Technology, 2018, 96(5-8): 2791-2801.

[34] Shevchik S A, Kenel C, Leinenbach C, et al. Acoustic emission for in situ quality monitoring in additive manufacturing using spectral convolutional neural networks. Additive Manufacturing, 2018, 21: 598-604.

[35] Grasso M, Demir A G, Previtali B, et al. In situ monitoring of selective laser melting of zinc powder via infrared imaging of the process plume. Robotics and Computer-Integrated Manufacturing, 2018, 49: 229-239.

[36] Grasso M, Colosimo B M. A statistical learning method for image-based monitoring of the plume signature in laser powder bed fusion. Robotics and Computer-Integrated Manufacturing, 2019, 57: 103-115.

[37] Repossini G, Laguzza V, Grasso M, et al. On the use of spatter signature for in-situ monitoring of laser powder bed fusion. Additive Manufacturing, 2017, 16: 35-48.

[38] Grasso M, Laguzza V, Semeraro Q, et al. In-process monitoring of selective laser melting: spatial detection of defects via image data analysis. Journal of Manufacturing Science and Engineering, 2017, 139(5): 051001-1-051001-16.

[39] Colosimo B M, Grasso M. Spatially weighted PCA for monitoring video image data with application to additive manufacturing. Journal of Quality Technology, 2018, 50(4): 391-417.

[40] Dunbar A J, Denlinger E R, Heigel J, et al. Development of experimental method for in situ distortion and temperature measurements during the laser powder bed fusion additive manufacturing process. Additive Manufacturing, 2016, 12: 25-30.

[41] Lane B, Moylan S, Whitenton E P, et al. Thermographic measurements of the commercial laser powder bed fusion process at NIST. Rapid Prototyping Journal, 2016, 22(5): 778-787.

[42] Mireles J, Ridwan S, Morton P A, et al. Analysis and correction of defects within parts fabricated using powder bed fusion technology. Surface Topography: Metrology and Properties, 2015, 3(3): 34002.

[43] Land W S, Zhang B, Ziegert J, et al. In-situ metrology system for laser powder bed fusion additive process. Procedia Manufacturing, 2015, 1: 393-403.

[44] Krauss H, Zeugner T, Zaeh M F. Layerwise monitoring of the selective laser melting process by thermography. Physics Procedia, 2014, 56: 64-71.

[45] Clijsters S, Craeghs T, Buls S, et al. In situ quality control of the selective laser melting process using a high-speed, real-time melt pool monitoring system. The International Journal of Advanced Manufacturing Technology, 2014, 75(5-8): 1089-1101.

[46] Furumoto T, Alkahari M R, Ueda T, et al. Monitoring of laser consolidation process of metal powder with high speed video camera. Physics Procedia, 2012, 39: 760-766.

[47] Jacobsmühlen J, Kleszczynski S, Schneider D, et al. High resolution imaging for inspection of laser beam melting systems. In: Proc. Instrumentation and Measurement Technology Conference, IEEE, 2013. 
[48] Alkahari M R, Furumoto T, Ueda T, et al. Melt pool and single track formation in selective laser sintering/selective laser melting. Advanced Materials Research, 2014, 933: 196-201.

[49] Berumen S, Bechmann F, Lindner S, et al. Quality control of laser- and powder bed-based additive manufacturing (AM) technologies. Physics Procedia, 2010, 5: 617-622.

[50] Jacobsmühlen J, Kleszczynski S, Witt G, et al. Elevated region area measurement for quantitative analysis of laser beam melting process stability. In: Proceedings of the 26th Solid Freeform Fabrication (SFF) Symposium, 2015.

[51] Kleszczynski S, Jacobsmühlen J Z, Sehr J T. et al. Error detection in laser beam melting systems by high resolution imaging. In: Proceedings of the 23rd Solid Freeform Fabrication Symposium, 2012.

[52] Kleszczynski S, Jacobsmühlen J Z, Reinarz B, et al. Improving process stability of laser beam melting systems. In: Proc. Fraunhofer Direct Digital Manufacturing Conference, 2014.

[53] Rieder H, Dillhöfer A, Spies M, et al. Online monitoring of additive manufacturing processes using ultrasound. In: Proc. 11th European Conference on Non-Destructive Testing (ECNDT 2014), Prague, Czech Republic, 2014: 6-10.

[54] Thombansen U, Gatej A, Pereira M. Process observation in fiber laser-based selective laser melting. Optical Engineering 2015, 54(1): 011008.

[55] Zenzinger G, Bamberg J, Ladewig A, et al. Process monitoring of additive manufacturing by using optical tomography. In: AIP Conference Proceedings, American Institute of Physics, 2015, 1650(1): 164-170.

[56] McNeil J L, Sisco K, Frederick C, et al. In-situ monitoring for defect identification in nickel alloy complex geometries fabricated by L-PBF additive manufacturing. Metallurgical and materials transactions, A, 2020, 51(12): 6528.

[57] Raplee J, Plotkowski A, Kirka M M, et al. Thermographic microstructure monitoring in electron beam additive manufacturing. Scientific Reports, 2017, doi: 10.1038/ srep43554.

[58] Foster S J, Carver K, Dinwiddie R B, et al. Process-defectstructure-property correlations during laser powder bed fusion of alloy 718: Role of in situ and ex situ characterizations. Metallurgical and Materials Transactions A, 2018, 49(11): 5775-5798.

[59] Forien J B, Calta N P, DePond P J, et al. Detecting keyhole pore defects and monitoring process signatures during laser powder bed fusion: A correlation between in situ pyrometry and ex situ X-ray radiography. Additive Manufacturing, 2020, 35: 101336.

[60] Zouhri W, Dantan J Y, Häfner B, et al. Optical process monitoring for Laser-Powder Bed Fusion (L-PBF). CIRP Journal of Manufacturing Science and Technology, 2020, 31: 607-617.

[61] Ji Z, Han Q. A novel image feature descriptor for SLM spattering pattern classification using a consumable camera. The International Journal of Advanced Manufacturing Technology, 2020, 110(11-12): 2955-2976.

[62] Scime L, Beuth J. Anomaly detection and classification in a laser powder bed additive manufacturing process using a trained computer vision algorithm. Additive Manufacturing, 2018, 19: 114-126.

[63] Scime L, Beuth J. A multi-scale convolutional neural network for autonomous anomaly detection and classification in a laser powder bed fusion additive manufacturing process. Additive Manufacturing, 2018, 24: 273-286.
[64] Scime L, Fisher B, Beuth J. Using coordinate transforms to improve the utility of a fixed field of view high speed camera for additive manufacturing applications. Manufacturing Letters, 2018, 15: 104-106.

[65] Scime L, Beuth J. Melt pool geometry and morphology variability for the Inconel 718 alloy in a laser powder bed fusion additive manufacturing process. Additive Manufacturing, 2019, 29: 100830.

[66] Scime L, Beuth J. Using machine learning to identify in-situ melt pool signatures indicative of flaw formation in a laser powder bed fusion additive manufacturing process. Additive Manufacturing, 2019, 25: 151-165.

[67] Baumgartl H, Tomas J, Buettner R, et al. A deep learningbased model for defect detection in laser-powder bed fusion using in-situ thermographic monitoring. Progress in Additive Manufacturing, 2020, 5(3): 277-285.

[68] Yang L, Lo L, Ding S, et al. Monitoring and detection of meltpool and spatter regions in laser powder bed fusion of super alloy Inconel 625. Progress in Additive Manufacturing, 2020, 5(4): 367-378.

[69] Peng $X$, Kong $L B$, Chen $Y$, et al. Design of a multi-sensor monitoring system for additive manufacturing process. Nanomanufacturing and Metrology, 2020, 3(2): 142-150.

[70] Lu Q Y, Nguyen N V, Hum A J W, et al. Optical in-situ monitoring and correlation of density and mechanical properties of stainless steel parts produced by selective laser melting process based on varied energy density. Journal of Materials Processing Technology, 2019, 271: 520-531.

[71] Lu Q Y, Nguyen N V, Hum A J W, et al. Identification and evaluation of defects in selective laser melted $316 \mathrm{~L}$ stainless steel parts via in-situ monitoring and micro computed tomography. Additive Manufacturing, 2020, 35: 101287.

[72] Lough C S, Wang X, Landers R G, et al. In-situ local part qualification of SLM 304L stainless steel through voxel based processing of SWIR imaging data. Measurements, 2019, 12: 14.

[73] Lough C S, Wang X, Smith C C, et al. Correlation of SWIR imaging with LPBF $304 \mathrm{~L}$ stainless steel part properties. Additive Manufacturing, 2020, 35: 101359.

[74] Lough C S, Escano L I, Qu M, et al. In-situ optical emission spectroscopy of selective laser melting. Journal of Manufacturing Processes, 2020, 53: 336-341.

[75] Krauss H, Eschey C, Zaeh M. Thermography for monitoring the selective laser melting process. In: Proceedings of the 23rd Solid Freeform Fabrication Symposium, 2012: 999-1014.

[76] Williams R J, Piglione A, Rønneberg T, et al. In situ thermography for laser powder bed fusion: Effects of layer temperature on porosity, microstructure and mechanical properties. Additive Manufacturing, 2019, 30: 100880.

[77] Okaro I A, Jayasinghe S, Sutcliffe C, et al. Automatic fault detection for laser powder-bed fusion using semi-supervised machine learning. Additive Manufacturing, 2019, 27: 42-53.

[78] Gutknecht K, Haferkamp L, Cloots M, et al. Determining process stability of laser powder bed fusion using pyrometry. Procedia CIRP, 2020, 95: 127-132.

[79] N N. Neue Möglichkeiten mit 3D (New possibilities in 3D). Werkzeug \& Formenbau, 2015, 9(4): 64-66. (In German)

[80] Colosimo B M, Grasso M. In-situ monitoring in L-PBF: Opportunities and challenges. Procedia CIRP, 2020, 94: 388391.

[81] N N. Positionsbezogene Echtzeitüberwachung und dreidimensionale Visualisierung (Position-correlated real-time monitoring and three-dimensional visualization). WOMag, 2015(9): 1-3. (In German) 